\title{
Asymptotics near $\pm m$ of the spectral shift function for Dirac operators with non-constant magnetic fields
}

\author{
Rafael Tiedra de Aldecoa* \\ Facultad de Matemáticas, Pontificia Universidad Católica de Chile, \\ Av. Vicuña Mackenna 4860, Santiago, Chile \\ E-mail: rtiedra@mat.puc.cl
}

\begin{abstract}
We consider a 3-dimensional Dirac operator $H_{0}$ with non-constant magnetic field of constant direction, perturbed by a sign-definite matrix-valued potential $V$ decaying fast enough at infinity. Then we determine asymptotics, as the energy goes to $+m$ and $-m$, of the spectral shift function for the pair $\left(H_{0}+V, H_{0}\right)$. We obtain, as a by-product, a generalised version of Levinson's Theorem relating the eigenvalues asymptotics of $H_{0}+V$ near $+m$ and $-m$ to the scattering phase shift for the pair $\left(H_{0}+V, H_{0}\right)$.
\end{abstract}

\section{Introduction}

It is known [38] that the free Dirac Hamiltonian $H_{m}$ acting in the Hilbert space $\mathcal{H}:=\mathrm{L}^{2}\left(\mathbb{R}^{3} ; \mathbb{C}^{4}\right)$ is unitarily equivalent to the operator $h(P) \oplus-h(P)$, where $P:=-i \nabla$ and $\mathbb{R}^{3} \ni \xi \mapsto h(\xi):=\left(\xi^{2}+m^{2}\right)^{1 / 2}$. For this reason, the set $\{ \pm m\}=h\left[(\nabla h)^{-1}(\{0\})\right]$ of critical values of $h$ plays an important role in spectral analysis and scattering theory for Dirac operators. For instance, one cannot prove at $\pm m$ the usual limiting absorption principle for operators $H_{m}+V$, even with $V$ a regular perturbation of $H_{m}$, by using standard commutator methods. Both the statements and the proofs have to be modified (see e.g. $[4,19]$ ).

In this paper, we provide a new account on the spectral analysis of Dirac operators at the critical values by discussing the behaviour at $\pm m$ of the spectral shift function associated to sign-definite perturbations of Dirac operators with non-constant magnetic fields. Our work is closely related to [27] where G. D. Raikov treats a similar issue in the case of magnetic Pauli operators. It can also be considered as a complement of [33], where general properties of the spectrum of Dirac operators with variable magnetic fields of constant direction and matrix perturbations are determined. Other related results on the spectrum of 3-dimensional magnetic Dirac operators can be found in $[2,3,5,7,11,13,15,16,17,20,23,34,36,37]$.

Let us describe the content of this paper. We consider a relativistic spin- $\frac{1}{2}$ particle evolving in $\mathbb{R}^{3}$ in presence of a variable magnetic field of constant direction. By virtue of the Maxwell equations, we may assume with no loss of generality that the magnetic field has the form

$$
\vec{B}\left(x_{1}, x_{2}, x_{3}\right)=\left(0,0, b\left(x_{1}, x_{2}\right)\right) .
$$

The system is described in $\mathcal{H}$ by the Dirac operator

$$
H_{0}:=\alpha_{1} \Pi_{1}+\alpha_{2} \Pi_{2}+\alpha_{3} P_{3}+\beta m,
$$

* Supported by the Fondecyt Grant 1090008 and by the Iniciativa Cientifica Milenio ICM P07-027-F "Mathematical Theory of Quantum and Classical Magnetic Systems". 
where $\beta \equiv \alpha_{0}, \alpha_{1}, \alpha_{2}, \alpha_{3}$ are the usual Dirac-Pauli matrices, $m>0$ is the mass of the particle and $\Pi_{j}:=$ $-i \partial_{j}-a_{j}$ are the generators of the magnetic translations with a vector potential

$$
\vec{a}\left(x_{1}, x_{2}, x_{3}\right)=\left(a_{1}\left(x_{1}, x_{2}\right), a_{2}\left(x_{1}, x_{2}\right), 0\right)
$$

that satisfies $b=\partial_{1} a_{2}-\partial_{2} a_{1}$. Since $a_{3}=0$, we write $P_{3}=-i \partial_{3}$ instead of $\Pi_{3}$. We assume that the function $b: \mathbb{R}^{2} \rightarrow \mathbb{R}$ is continuous (see Section 2 for details), so that $H_{0}$, defined on $C_{0}^{\infty}\left(\mathbb{R}^{3} ; \mathbb{C}^{4}\right)$, can be extended uniquely to a selfadjoint operator in $\mathcal{H}$ with domain $\mathcal{D}\left(H_{0}\right)$.

Then we consider a bounded positive multiplication operator $V \in C\left(\mathbb{R}^{3} ; \mathscr{B}_{\mathrm{h}}\left(\mathbb{C}^{4}\right)\right)$, where $\mathscr{B}_{\mathrm{h}}\left(\mathbb{C}^{4}\right)$ is the set of $4 \times 4$ hermitian matrices, and define the perturbed Hamiltonian $H_{ \pm}:=H_{0} \pm V$. Since $V$ is bounded and symmetric, the operator $H_{ \pm}$is selfadjoint in $\mathcal{H}$ and has domain $\mathcal{D}(H)=\mathcal{D}\left(H_{0}\right)$. We also assume that $|V(x)|$ decays more rapidly than $|x|^{-3}$ as $|x| \rightarrow \infty$ and that

$$
\left(H_{ \pm}-z\right)^{-3}-\left(H_{0}-z\right)^{-3} \in S_{1}(\mathcal{H}) \quad \text { for each } \quad z \in \mathbb{R} \backslash\left\{\sigma\left(H_{0}\right) \cup \sigma\left(H_{ \pm}\right)\right\},
$$

where $S_{1}(\mathcal{H})$ denotes the set of trace class operators in $\mathcal{H}$.

Under these assumptions, there exists a unique function $\xi\left(\cdot ; H_{ \pm}, H_{0}\right) \in \mathrm{L}^{1}\left(\mathbb{R} ;(1+|\lambda|)^{-4} \mathrm{~d} \lambda\right)$ such that the Lifshits-Krein trace formula

$$
\operatorname{Tr}\left[f\left(H_{ \pm}\right)-f\left(H_{0}\right)\right]=\int_{\mathbb{R}} \mathrm{d} \lambda f^{\prime}(\lambda) \xi\left(\lambda ; H_{ \pm}, H_{0}\right)
$$

holds for each $f \in C_{0}^{\infty}(\mathbb{R})$ (see [39, Sec. 8.11]). The function $\xi\left(\cdot ; H_{ \pm}, H_{0}\right)$ is called the spectral shift function for the pair $\left(H_{ \pm}, H_{0}\right)$. It vanishes identically on $\mathbb{R} \backslash\left\{\sigma\left(H_{0}\right) \cup \sigma\left(H_{ \pm}\right)\right\}$, and can be related to the number of eigenvalues of $H_{ \pm}$in $(-m, m)$ (see Remark 4.5). Morever, for almost every $\lambda \in \sigma_{\mathrm{ac}}\left(H_{0}\right)$ the spectral shift function is related to the scattering matrix $S\left(\lambda ; H_{ \pm}, H_{0}\right)$ for the pair $\left(H_{ \pm}, H_{0}\right)$ by the Birman-Krein formula

$$
\operatorname{det} S\left(\lambda ; H_{ \pm}, H_{0}\right)=\mathrm{e}^{-2 \pi i \xi\left(\lambda ; H_{ \pm}, H_{0}\right)} .
$$

After identification of $\xi\left(\cdot ; H_{ \pm}, H_{0}\right)$ with some representative of its equivalence class, our results are the following. In Proposition 4.4, we show that there exists a constant $\zeta>0$ defined in terms of $b$ (cf. Proposition 2.1) such that $\xi\left(\cdot ; H_{ \pm}, H_{0}\right)$ is bounded on each compact subset of $\left(-\sqrt{m^{2}+\zeta}, \sqrt{m^{2}+\zeta}\right) \backslash\{ \pm m\}$ and is continuous on $\left(-\sqrt{m^{2}+\zeta}, \sqrt{m^{2}+\zeta}\right) \backslash\left(\{ \pm m\} \cup \sigma_{\mathrm{p}}\left(H_{ \pm}\right)\right)$. In Theorem 6.5, we determine the asymptotic behaviour of $\xi\left(\lambda ; H_{ \pm}, H_{0}\right)$ as $\lambda \rightarrow \pm m,|\lambda|<m$, and in Theorem 6.14, we determine the asymptotic behaviour of $\xi\left(\lambda ; H_{ \pm}, H_{0}\right)$ as $\lambda \rightarrow \pm m,|\lambda|>m$. In both cases, one has $\xi\left(\lambda ; H_{ \pm}, H_{0}\right) \rightarrow \pm \infty$ as $\lambda \rightarrow \mp m$. The divergence of $\xi\left(\lambda ; H_{ \pm}, H_{0}\right)$ near $\lambda= \pm m$ scales as the number of eigenvalues near 0 of certain BerezinToeplitz type operators. When $V$ admits a power-like or exponential decay at infinity, or when it has a compact support, we give the first term of the asymptotic expansion of $\xi\left(\lambda, ; H_{ \pm}, H_{0}\right)$ near $\lambda= \pm m$ (see Proposition 6.10 and Corollary 6.17). In these cases, we also show that the limits

$$
\lim _{\varepsilon \searrow 0} \frac{\xi\left(m+\varepsilon ; H_{-}, H_{0}\right)}{\xi\left(m-\varepsilon ; H_{-}, H_{0}\right)} \quad \text { and } \quad \lim _{\varepsilon \searrow 0} \frac{\xi\left(-m-\varepsilon ; H_{+}, H_{0}\right)}{\xi\left(-m+\varepsilon ; H_{+}, H_{0}\right)}
$$

exist and are equal to positive constants depending on the decay rate of $V$ at infinity (see Corollary 6.18 for a precise statement). This can be interpreted as a generalised version of Levinson's Theorem relating the eigenvalues asymptotics of $H_{ \pm}$near $\mp m$ to the scattering phase shift for the pair $\left(H_{ \pm}, H_{0}\right)$ (see [21, 22] for usual versions of Levinson's Theorem for Dirac operators). The relation between the behaviour of the spectral shift function near $\lambda=+m$ and near $\lambda=-m$ is explained in Remark 6.15 by using the charge conjugation symmetry.

These results are consistent with the results of [27] (where Pauli operators with non-constant magnetic fields are considered) and [12] (where Schrödinger operators with constant magnetic field are considered). Part of the interest of this work relies on the fact that we were able to exhibit a non-trivial class of matrix potentials $V$ satisfying (1.1), even though $H_{0}$ is not a bounded perturbation of the free Dirac operator. We refer to Remark 3.3 and Section 7 for a discussion of this issue.

Let us fix the notations that are used in the paper. The norm and scalar product of $\mathcal{H} \equiv \mathrm{L}^{2}\left(\mathbb{R}^{3} ; \mathbb{C}^{4}\right)$ are denoted by $\|\cdot\|$ and $\langle\cdot, \cdot\rangle$. The symbol $\otimes$ stands for the closed tensor product of Hilbert spaces and $S_{p}(\mathcal{H})$, 
$p \in[1, \infty]$, denotes the $p$-th Schatten-von Neumann class of operators in $\mathcal{H}\left(S_{\infty}(\mathcal{H})\right.$ is the set of compact operators in $\mathcal{H}$ ). We denote by $\|\cdot\|_{p}$ the corresponding operator norm. The variable $x \in \mathbb{R}^{3}$ is often written as $x \equiv\left(x_{\perp}, x_{3}\right)$, with $x_{\perp} \in \mathbb{R}^{2}$ and $x_{3} \in \mathbb{R}$. The symbol $Q_{j}, j=1,2,3$, denotes the multiplication operator by $x_{j}$ in $\mathcal{H}, Q:=\left(Q_{1}, Q_{2}, Q_{3}\right)$, and $Q_{\perp}:=\left(Q_{1}, Q_{2}\right)$. Sometimes, when the context is unambiguous, we consider the operators $Q_{j}$ and $P_{j}$ as operators in $\mathrm{L}^{2}(\mathbb{R})$ instead of $\mathcal{H}$ without changing the notations. Given a selfadjoint operator $A$ in a Hilbert space $\mathcal{G}$, the symbol $E^{A}(\cdot)$ stands for the spectral measure of $A$.

\section{Unperturbed operator}

Throughout this paper we assume that the component $b: \mathbb{R}^{2} \rightarrow \mathbb{R}$ of the magnetic field $\vec{B} \equiv(0,0, b)$ belongs to the class of "admissible" magnetic fields defined in [27, Sec. 2.1]. Namely, we assume that $b=b_{0}+\widetilde{b}$, where $b_{0}>0$ is a constant while the function $\widetilde{b}: \mathbb{R}^{2} \rightarrow \mathbb{R}$ is such that the Poisson equation

$$
\Delta \widetilde{\varphi}=\widetilde{b}
$$

admits a solution $\widetilde{\varphi}: \mathbb{R}^{2} \rightarrow \mathbb{R}$, continuous and bounded together with its derivatives of order up to two. We also define $\varphi_{0}\left(x_{\perp}\right):=\frac{1}{4} b_{0}\left|x_{\perp}\right|^{2}$ for each $x_{\perp} \in \mathbb{R}^{2}$ and $\operatorname{set} \varphi:=\varphi_{0}+\widetilde{\varphi}$. Then we obtain a vector potential $\vec{a} \equiv\left(a_{1}, a_{2}, 0\right) \in C^{1}\left(\mathbb{R}^{2} ; \mathbb{R}^{3}\right)$ for the magnetic field $\vec{B}$ by putting

$$
a_{1}:=-\partial_{2} \varphi \quad \text { and } \quad a_{2}:=\partial_{1} \varphi .
$$

(changing, if necessary, the gauge, we shall always assume that the vector potential $\vec{a}$ is of this form). We refer to [27] for further properties and examples of admissible magnetic fields.

Since the vector potential $\vec{a}$ belongs to $L_{\text {loc }}^{\infty}\left(\mathbb{R}^{2} ; \mathbb{R}^{3}\right)$, the magnetic Dirac operator

$$
H_{0}=\alpha_{1} \Pi_{1}+\alpha_{2} \Pi_{2}+\alpha_{3} P_{3}+\beta m
$$

satisfies all the properties of [33, Sec. 2.1]. The operator $H_{0}$ is essentially selfadjoint on $C_{0}^{\infty}\left(\mathbb{R}^{3} ; \mathbb{C}^{4}\right)$, with domain $\mathcal{D}\left(H_{0}\right) \subset \mathcal{H}_{\text {loc }}^{1 / 2}\left(\mathbb{R}^{3} ; \mathbb{C}^{4}\right)$, the spectrum of $H_{0}$ satisfies

$$
\sigma\left(H_{0}\right)=\sigma_{\mathrm{ac}}\left(H_{0}\right)=\left(-\infty,-\mu_{0}\right] \cup\left[\mu_{0}, \infty\right),
$$

and we have the identity

$$
H_{0}^{2}=\left(\begin{array}{cccc}
H_{\perp}^{-} \otimes 1+1 \otimes\left(P_{3}^{2}+m^{2}\right) & 0 & 0 & 0 \\
0 & H_{\perp}^{+} \otimes 1+1 \otimes\left(P_{3}^{2}+m^{2}\right) & 0 & 0 \\
0 & 0 & H_{\perp}^{-} \otimes 1+1 \otimes\left(P_{3}^{2}+m^{2}\right) & 0 \\
0 & 0 & 0 & H_{\perp}^{+} \otimes 1+1 \otimes\left(P_{3}^{2}+m^{2}\right)
\end{array}\right)
$$

with respect to the tensorial decomposition $\mathrm{L}^{2}\left(\mathbb{R}^{2}\right) \otimes \mathrm{L}^{2}(\mathbb{R})$ of $\mathrm{L}^{2}\left(\mathbb{R}^{3}\right)$. Here the operators $H_{\perp}^{ \pm}$are the components of the Pauli operator $H_{\perp}:=H_{\perp}^{-} \oplus H_{\perp}^{+}$in $\mathrm{L}^{2}\left(\mathbb{R}^{2} ; \mathbb{C}^{2}\right)$ associated with the vector potential $\left(a_{1}, a_{2}\right)$, and $\mu_{0}:=\sqrt{\inf \sigma\left(H_{\perp}\right)+m^{2}}$.

We recall from [27, Sec. 2.2] that $\operatorname{dim} \operatorname{ker}\left(H_{\perp}^{-}\right)=\infty$, that $\operatorname{dim} \operatorname{ker}\left(H_{\perp}^{+}\right)=0$ and that we have the following result.

Proposition 2.1. Let $b$ be an admissible magnetic field with $b_{0}>0$. Then $0=\inf \sigma\left(H_{\perp}\right)$ is an isolated eigenvalue of infinite multiplicity. More precisely, we have

$$
\operatorname{dim} \operatorname{ker}\left(H_{\perp}\right)=\infty \quad \text { and } \quad(0, \zeta) \subset \mathbb{R} \backslash \sigma\left(H_{\perp}\right),
$$

where

$$
\zeta:=2 b_{0} \mathrm{e}^{-2 \operatorname{osc}(\widetilde{\varphi})} \quad \text { and } \quad \operatorname{osc}(\widetilde{\varphi}):=\sup _{x_{\perp} \in \mathbb{R}^{2}} \widetilde{\varphi}\left(x_{\perp}\right)-\inf _{x_{\perp} \in \mathbb{R}^{2}} \widetilde{\varphi}\left(x_{\perp}\right) .
$$

In particular, we have by Equation (2.1)

$$
\sigma\left(H_{0}\right)=\sigma_{\mathrm{ac}}\left(H_{0}\right)=(-\infty,-m] \cup[m, \infty) .
$$


Finally, since $(0, \zeta) \subset \mathbb{R} \backslash \sigma\left(H_{\perp}\right)$, we know from [33, Thm. 1.2.(d)] that the limits

$$
\lim _{\varepsilon \searrow 0}\left\langle Q_{3}\right\rangle^{-\nu_{3} / 2}\left(H_{0}-\lambda \mp i \varepsilon\right)^{-1}\left\langle Q_{3}\right\rangle^{-\nu_{3} / 2}, \quad \nu_{3}>1,
$$

exist for each $\lambda \in\left(-\sqrt{m^{2}+\zeta}, \sqrt{m^{2}+\zeta}\right) \backslash\{ \pm m\}$ (note that we use the usual notation $\langle\cdot\rangle:=\sqrt{1+|\cdot|^{2}}$ ).

\section{Perturbed operator}

We consider now the perturbed operators $H_{ \pm}=H_{0} \pm V$, where $V \equiv\left\{V_{j k}\right\}$ is the multiplication operator associated to the following matrix-valued function $V$.

Assumption 3.1. The function $V \in C\left(\mathbb{R}^{3} ; \mathscr{B}_{\mathrm{h}}\left(\mathbb{C}^{4}\right)\right)$ satisfies for each $x \equiv\left(x_{\perp}, x_{3}\right) \in \mathbb{R}^{3}$ and each $j, k \in$ $\{1, \ldots, 4\}$

$$
V(x) \geq 0 \quad \text { and } \quad\left|V_{j k}(x)\right| \leq \text { Const. }\left\langle x_{\perp}\right\rangle^{-\nu_{\perp}}\left\langle x_{3}\right\rangle^{-\nu_{3}} \quad \text { for some } \nu_{\perp}>2 \text { and } \nu_{3}>1 .
$$

The potential $V$ in Assumption 3.1 is short-range along $x_{3}$. So we know from [33, Thm. 1.2] that

(i) $\sigma_{\text {ess }}\left(H_{ \pm}\right)=\sigma_{\text {ess }}\left(H_{0}\right)=(-\infty,-m] \cup[m, \infty)$.

(ii) The point spectrum of $H_{ \pm}$in $\left(-\sqrt{m^{2}+\zeta}, \sqrt{m^{2}+\zeta}\right) \backslash\{ \pm m\}$ is composed of eigenvalues of finite multiplicity and with no accumulation point.

(iii) $H_{ \pm}$has no singular continuous spectrum in $\left(-\sqrt{m^{2}+\zeta}, \sqrt{m^{2}+\zeta}\right)$. In particular, $H_{0}$ and $H_{ \pm}$have a common spectral gap in $(-m, m)$.

Using the formula

$$
(A+\lambda)^{-\gamma}=\Gamma(\gamma)^{-1} \int_{0}^{\infty} \mathrm{d} t t^{\gamma-1} \mathrm{e}^{-t(A+\lambda)}, \quad A: \mathcal{D}(A) \rightarrow \mathcal{H}, A \geq 0, \lambda, \gamma>0,
$$

the diamagnetic inequality [1, Thm. 2.3], and the compactness criterion [9, Thm. 5.7.1], we find that

$$
\left|V_{j k}\right|^{1 / 2}\left(\sum_{\ell \leq 3} \Pi_{\ell}^{*} \Pi_{\ell}+m^{2}\right)^{-1 / 4} \in S_{\infty}\left[\mathrm{L}^{2}\left(\mathbb{R}^{3}\right)\right] .
$$

Since $b$ is bounded this implies that

$$
\left|H_{0}\right|^{-1 / 2} V\left|H_{0}\right|^{-1 / 2} \leq\left|H_{0}\right|^{-1 / 2}\left(\sum_{j, k \leq 4}\left|V_{j k}\right|\right)\left|H_{0}\right|^{-1 / 2} \in S_{\infty}(\mathcal{H}) .
$$

So $\left|H_{0}\right|^{-1 / 2} V\left|H_{0}\right|^{-1 / 2}$ also belongs to $S_{\infty}(\mathcal{H})$, since $S_{\infty}(\mathcal{H})$ is an hereditary $C^{*}$-subalgebra of $\mathscr{B}(\mathcal{H})$ [24, Cor. 3.2.3]. One has in particular

$$
V^{1 / 2}\left(\left|H_{0}\right|+1\right)^{-1 / 2} \in S_{\infty}(\mathcal{H}) .
$$

The standard criterion [31, Thm. XI.20] shows that

$$
\left|V_{j k}\right|^{1 / 2}\left(-\Delta+m^{2}\right)^{-\gamma} \in S_{q}\left[\mathrm{~L}^{2}\left(\mathbb{R}^{3}\right)\right] \quad \text { if } q \in[2, \infty) \text { and } \gamma q>3 / 2 .
$$

This together with arguments as above implies that

$$
V^{1 / 2}\left|H_{0}\right|^{-\gamma} \in S_{q}(\mathcal{H}) \quad \text { if } q \geq 2 \text { is even and } \gamma q>3 .
$$

So we have in particular that

$$
V^{1 / 2} E^{H_{0}}(B) \in S_{2}(\mathcal{H}) \quad \text { for any bounded borel set } B \subset \mathbb{R} .
$$

In the sequel we shall need a more restrictive assumption on $V$. For this, we recall that there exists numbers $z \in \mathbb{R} \backslash\left\{\sigma\left(H_{0}\right) \cup \sigma\left(H_{ \pm}\right)\right\}$since $H_{0}$ and $H_{ \pm}$have a common spectral gap in $(-m, m)$. We also set $R_{0}(z):=$ $\left(H_{0}-z\right)^{-1}$ and $R_{ \pm}(z):=\left(H_{ \pm}-z\right)^{-1}$ for $z \in \mathbb{C} \backslash \sigma\left(H_{0}\right)$ and $z \in \mathbb{C} \backslash \sigma\left(H_{ \pm}\right)$, respectively. 
Assumption 3.2. The function $V \in C\left(\mathbb{R}^{3} ; \mathscr{B}_{\mathrm{h}}\left(\mathbb{C}^{4}\right)\right)$ satisfies for each $x \in \mathbb{R}^{3}$ and each $j, k \in\{1, \ldots, 4\}$

$$
V(x) \geq 0 \quad \text { and } \quad\left|V_{j k}(x)\right| \leq \text { Const. }\langle x\rangle^{-\nu} \text { for some constant } \nu>3 .
$$

Furthermore, $V$ is chosen such that

$$
R_{ \pm}^{3}(z)-R_{0}^{3}(z) \in S_{1}(\mathcal{H}) \quad \text { for each } z \in \mathbb{R} \backslash\left\{\sigma\left(H_{0}\right) \cup \sigma\left(H_{ \pm}\right)\right\} .
$$

Note that (3.5) implies (3.1) if one takes $\nu_{3} \in(1, \nu-2)$ and $\nu_{\perp}:=\nu-\nu_{3}$. Note also that the choice of function $\lambda \mapsto(\lambda-z)^{-3}$ in the trace class condition (3.6) has been made for convenience. Many other choices would also guarantee the existence of the spectral shift function for the pair $\left(H_{ \pm}, H_{0}\right)$ (see e.g. [39, Sec. 8.11]).

Remark 3.3. Since the operator $H_{0}$ is not a bounded perturbation of the free Dirac operator, we cannot apply the results of [40, Sec. 4] to prove the inclusion (3.6) under the condition (3.5). In general, one has to impose additional assumptions on $V$ to get the result. For instance, if $V$ verifies (3.5), and

(i) $\left[V, \alpha_{1}\right]=\left[V, \alpha_{2}\right]=0$,

(ii) for each $x \in \mathbb{R}^{3}$ and each $j, k, \ell \in\{1, \ldots, 4\}$, one has $\left|\left(\partial_{\ell} V_{j k}\right)(x)\right| \leq$ Const. $\langle x\rangle^{-\varsigma}$ for some $\varsigma>3$,

(iii) for each $j, k, \ell \in\{1, \ldots, 4\}$, one has $\left(\partial_{\ell} \partial_{3} V_{j k}\right) \in \mathrm{L}^{\infty}\left(\mathbb{R}^{3}\right)$,

then (3.6) is satisfied. Furthermore, if $V$ is scalar, then the same is true without assuming (iii) (and (i) is trivially satisfied). The proof of these statements can be found in the appendix. Here, we only note that a matrix $\mathrm{V} \in \mathscr{B}_{\mathrm{h}}\left(\mathbb{C}^{4}\right)$ satisfying $(i)$ is necessarily of the form

$$
\mathrm{V}=\left(\begin{array}{cccc}
\mathrm{v}_{1} & 0 & \mathrm{v}_{3} & 0 \\
0 & \mathrm{v}_{2} & 0 & \mathrm{v}_{3} \\
\mathrm{v}_{3} & 0 & \mathrm{v}_{2} & 0 \\
0 & \mathrm{v}_{3} & 0 & \mathrm{v}_{1}
\end{array}\right),
$$

with $\mathrm{v}_{1}, \mathrm{v}_{2} \in \mathbb{R}$ and $\mathrm{v}_{3} \in \mathbb{C}$.

\section{Spectral shift function}

In this section we recall some results due to A. Pushnitski on the representation of the spectral shift function for a pair of not semibounded selfadjoint operators.

Given a Lebesgue measurable set $B \subset \mathbb{R}$, we define $\mu(B):=\frac{1}{\pi} \int_{B} \frac{\mathrm{d} t}{1+t^{2}}$, and note that $\mu(\mathbb{R})=1$. Furthermore, if $T=T^{*}$ is a compact operator in a separable Hilbert space $\mathcal{G}$, we set

$$
n_{ \pm}(s ; T):=\operatorname{rank} E^{ \pm T}((s, \infty)) \text { for } s>0 .
$$

Then we have the following estimates.

Lemma 4.1 (Lemma 2.1 of [26]). Let $T_{1}=T_{1}^{*} \in S_{\infty}(\mathcal{H})$ and $T_{2}=T_{2}^{*} \in S_{1}(\mathcal{H})$. Then one as for each $s_{1}, s_{2}>0$

$$
\int_{\mathbb{R}} \mathrm{d} \mu(t) n_{ \pm}\left(s_{1}+s_{2} ; T_{1}+t T_{2}\right) \leq n_{ \pm}\left(s_{1} ; T_{1}\right)+\frac{1}{\pi s_{2}}\left\|T_{2}\right\|_{1} .
$$

For $z \in \mathbb{C} \backslash \sigma\left(H_{0}\right)$, we define the usual weighted resolvent

$$
T(z):=V^{1 / 2}\left(H_{0}-z\right)^{-1} V^{1 / 2}
$$

and the corresponding real and imaginary parts

$$
A(z):=\operatorname{Re} T(z) \quad \text { and } \quad B(z):=\operatorname{Im} T(z) .
$$

Then the next lemma is direct consequence of the inclusions (3.2)-(3.4) and [25, Prop. 4.4.(i)]. 
Lemma 4.2. Let $V$ satisfy Assumption 3.1. Then, for almost every $\lambda \in \mathbb{R}$, the limits $A(\lambda+i 0):=\lim _{\varepsilon} \backslash 0 A(\lambda+$ $i \varepsilon)$ and $B(\lambda+i 0):=\lim _{\varepsilon \backslash 0} B(\lambda+i \varepsilon) \geq 0$ exist in $S_{4}(\mathcal{H})$.

Next theorem follows from the inclusions (3.2), (3.4), (3.6), from the equations (1.9), (8.1), (8.2) of [25], and from Theorem 8.1 of [25].

Theorem 4.3. Let $V$ satisfy Assumption 3.2. Then, for almost every $\lambda \in \mathbb{R}, \xi\left(\lambda ; H_{ \pm}, H_{0}\right)$ exists and is given by

$$
\xi\left(\lambda ; H_{ \pm}, H_{0}\right)= \pm \int_{\mathbb{R}} \mathrm{d} \mu(t) n_{\mp}(1 ; A(\lambda+i 0)+t B(\lambda+i 0)) .
$$

We know from (2.3) that $A(\lambda+i 0)$ and $B(\lambda+i 0)$ exist in $\mathscr{B}(\mathcal{H})$ for each $\lambda \in\left(-\sqrt{m^{2}+\zeta}, \sqrt{m^{2}+\zeta}\right) \backslash$ $\{ \pm m\}$. In Propositions 5.2-5.3 and Corollary 5.5 below we show that in fact $A(\lambda+i 0) \in S_{4}(\mathcal{H})$ and $B(\lambda+i 0) \in$ $S_{1}(\mathcal{H})$ for each $\lambda \in\left(-\sqrt{m^{2}+\zeta}, \sqrt{m^{2}+\zeta}\right) \backslash\{ \pm m\}$. Hence, by Lemma 4.1, the r.h.s. of (4.1) will turn out to be well-defined for every $\lambda \in\left(-\sqrt{m^{2}+\zeta}, \sqrt{m^{2}+\zeta}\right) \backslash\{ \pm m\}$. In the next proposition we state some regularity properties of the function

$$
\left(-\sqrt{m^{2}+\zeta}, \sqrt{m^{2}+\zeta}\right) \backslash\{ \pm m\} \ni \lambda \mapsto \widetilde{\xi}\left(\lambda ; H_{ \pm}, H_{0}\right):= \pm \int_{\mathbb{R}} \mathrm{d} \mu(t) n_{\mp}(1 ; A(\lambda+i 0)+t B(\lambda+i 0)) .
$$

The proof (which relies on Propositions 5.2-5.3, Lemma 5.4, Corollary 5.5 and the stability result [14, Thm. 3.12]) is similar to the one of [6, Sec. 4.2.1].

Proposition 4.4. Let $V$ satisfy Assumption 3.1. Then $\widetilde{\xi}\left(\cdot ; H_{ \pm}, H_{0}\right)$ is bounded on each compact subset of $\left(-\sqrt{m^{2}+\zeta}, \sqrt{m^{2}+\zeta}\right) \backslash\{ \pm m\}$ and is continuous on $\left(-\sqrt{m^{2}+\zeta}, \sqrt{m^{2}+\zeta}\right) \backslash\left(\{ \pm m\} \cup \sigma_{\mathrm{p}}\left(H_{ \pm}\right)\right)$.

In the sequel, we identify the functions $\widetilde{\xi}\left(\cdot ; H_{ \pm}, H_{0}\right)$ and $\xi\left(\cdot ; H_{ \pm}, H_{0}\right)$ since they are equal for almost every $\lambda \in \mathbb{R}$ due to Theorem 4.3 (see [35] for a study where the r.h.s. of (4.1) is directly treated as a definition of $\left.\xi\left(\lambda ; H_{ \pm}, H_{0}\right)\right)$.

Remark 4.5. In the interval $(-m, m), H_{0}$ has no spectrum and the spectrum of $H_{ \pm}$is purely discrete. Thus the spectral shift function $\xi\left(\cdot ; H_{ \pm}, H_{0}\right)$ can be related to the number of eigenvalues of $H_{ \pm}$as follows: for $\lambda_{1}, \lambda_{2} \in(-m, m) \backslash \sigma\left(H_{ \pm}\right)$with $\lambda_{1}<\lambda_{2}$, we have (see [25, Thm. 9.1])

$$
\xi\left(\lambda_{1} ; H_{ \pm}, H_{0}\right)-\xi\left(\lambda_{2} ; H_{ \pm}, H_{0}\right)=\operatorname{rank} E^{H_{ \pm}}\left(\left[\lambda_{1}, \lambda_{2}\right)\right) .
$$

\section{Decomposition of the weighted resolvent}

In this section we decompose the weighted resolvent

$$
T(z)=V^{1 / 2}\left(H_{0}-z\right) V^{1 / 2}, \quad z \in \mathbb{C} \backslash \sigma\left(H_{0}\right),
$$

into a sum $T(z)=T_{\text {div }}(z)+T_{\text {bound }}(z)$, where $T_{\text {div }}(z)$ (respectively $T_{\text {bound }}(z)$ ) corresponds to the diverging (respectively non-diverging) part of $T(z)$ as $z \rightarrow \pm m$. Then we estimate the behaviour, in suitable Schatten norms, of each term as $z \rightarrow \pm m$. We refer to [12, Sec. 4] and [27, Sec. 4.2] for similar approaches in the case of the Schrödinger and Pauli operators.

Let $a$ and $a^{*}$ be the closures in $L^{2}\left(\mathbb{R}^{2}\right)$ of the operators given by

$$
\mathrm{a} \varphi:=\left(\Pi_{1}-i \Pi_{2}\right) \varphi \quad \text { and } \quad \mathrm{a}^{*} \varphi:=\left(\Pi_{1}+i \Pi_{2}\right) \varphi,
$$

for $\varphi \in C_{0}^{\infty}\left(\mathbb{R}^{2}\right)$. Then one has (see [38, Sec. 5.5.2] and [28, Sec. 5])

$$
H_{0}=\left(\begin{array}{cccc}
m & 0 & 1 \otimes P_{3} & \mathrm{a} \otimes 1 \\
0 & m & \mathrm{a}^{*} \otimes 1 & -1 \otimes P_{3} \\
1 \otimes P_{3} & \mathrm{a} \otimes 1 & -m & 0 \\
\mathrm{a}^{*} \otimes 1 & -1 \otimes P_{3} & 0 & -m
\end{array}\right),
$$


with

$$
\operatorname{ker}\left(\mathrm{a}^{*}\right)=\operatorname{ker}\left(\mathrm{aa}^{*}\right)=\operatorname{ker}\left(H_{\perp}^{-}\right) \subset \mathrm{L}^{2}\left(\mathbb{R}^{2}\right) .
$$

Now, let

$$
P:=\left(\begin{array}{llll}
P & 0 & 0 & 0 \\
0 & 0 & 0 & 0 \\
0 & 0 & P & 0 \\
0 & 0 & 0 & 0
\end{array}\right)
$$

be the orthogonal projection onto the union of the eigenspaces of $H_{0}$ corresponding to the values $\lambda= \pm m$. Since $P \equiv p \otimes 1$ is the orthogonal projection onto $\operatorname{ker}\left(H_{\perp}^{-}\right) \otimes \mathrm{L}^{2}(\mathbb{R})$, the equations (5.1) and (5.2) imply that $H_{0}$ and $\mathrm{P}$ commute:

$$
H_{0}^{-1} \mathrm{P}=\mathrm{P} H_{0}^{-1} \text {. }
$$

In fact, by using (2.2) and (5.1), one gets for each $z \in \mathbb{C} \backslash \sigma\left(H_{0}\right)$ the equalities

$$
\begin{aligned}
& \left(H_{0}-z\right)^{-1} \mathrm{P} \\
& =\left(H_{0}+z\right)\left(H_{0}^{2}-z^{2}\right)^{-1} \mathrm{P} \\
& =\left[p \otimes R\left(z^{2}-m^{2}\right)\right]\left(\begin{array}{cccc}
(z+m) & 0 & 0 & 0 \\
0 & 0 & 0 & 0 \\
0 & 0 & (z-m) & 0 \\
0 & 0 & 0 & 0
\end{array}\right)+\left[p \otimes P_{3} R\left(z^{2}-m^{2}\right)\right]\left(\begin{array}{llll}
0 & 0 & 1 & 0 \\
0 & 0 & 0 & 0 \\
1 & 0 & 0 & 0 \\
0 & 0 & 0 & 0
\end{array}\right),
\end{aligned}
$$

where $R(z):=\left(P_{3}^{2}-z\right)^{-1}, z \in \mathbb{C} \backslash[0, \infty)$, is the resolvent of $P_{3}^{2}$ in $\mathrm{L}^{2}(\mathbb{R})$. This allows us to decompose $T(z)$ as $T(z)=T_{\operatorname{div}}(z)+T_{\text {bound }}(z)$, with

$$
\begin{aligned}
& T_{\text {div }}(z):=V^{1 / 2}\left[p \otimes R\left(z^{2}-m^{2}\right)\right]\left(\begin{array}{cccc}
(z+m) & 0 & 0 & 0 \\
0 & 0 & 0 & 0 \\
0 & 0 & (z-m) & 0 \\
0 & 0 & 0 & 0
\end{array}\right) V^{1 / 2}, \\
& T_{\text {bound }}(z):=V^{1 / 2}\left[p \otimes P_{3} R\left(z^{2}-m^{2}\right)\right]\left(\begin{array}{cccc}
0 & 0 & 1 & 0 \\
0 & 0 & 0 & 0 \\
1 & 0 & 0 & 0 \\
0 & 0 & 0 & 0
\end{array}\right) V^{1 / 2}+V^{1 / 2}\left(H_{0}-z\right)^{-1} \mathrm{P}^{\perp} V^{1 / 2} \quad\left(\mathrm{P}^{\perp}:=1-\mathrm{P}\right) \text {. }
\end{aligned}
$$

One may note that this decomposition of $T(z)$ differs slightly from the simpler decomposition

$$
T(z)=V^{1 / 2}\left(H_{0}-z\right) \mathrm{P} V^{1 / 2}+V^{1 / 2}\left(H_{0}-z\right) \mathrm{P}^{\perp} V^{1 / 2},
$$

since the first term in $T_{\text {bound }}(z)$ is associated to the projection $\mathrm{P}$ and not the projection $\mathrm{P}^{\perp}$. This choice is motivated by the will of distinguishing clearly the contribution $T_{\text {div }}(z)$, that diverge as $z \rightarrow \pm m$, from the contribution $T_{\text {bound }}(z)$, that stays bounded as $z \rightarrow \pm m$.

For $\lambda \in \mathbb{R} \backslash\{0\}$, we can define the boundary value $R(\lambda)$ of the resolvent $R(z)$ as the operator with convolution kernel $r_{\lambda}(\cdot)$, where

$$
\mathbb{R} \ni x_{3} \mapsto r_{\lambda}\left(x_{3}\right):= \begin{cases}\frac{\mathrm{e}^{-\sqrt{-\lambda}\left|x_{3}\right|}}{2 \sqrt{-\lambda}} & \text { if } \lambda<0, \\ \frac{i \mathrm{e}^{i \sqrt{\lambda}\left|x_{3}\right|}}{2 \sqrt{\lambda}} & \text { if } \lambda>0 .\end{cases}
$$

So, we can extend the definition of $T_{\text {div }}(\cdot)$ to the values $\lambda \in \mathbb{R} \backslash\{ \pm m\}$ :

$$
T_{\text {div }}(\lambda):=V^{1 / 2}\left[p \otimes R\left(\lambda^{2}-m^{2}\right)\right]\left(\begin{array}{cccc}
(\lambda+m) & 0 & 0 & 0 \\
0 & 0 & 0 & 0 \\
0 & 0 & (\lambda-m) & 0 \\
0 & 0 & 0 & 0
\end{array}\right) V^{1 / 2} .
$$

In the following proposition, we show that the trace norm of $T_{\operatorname{div}}(z)$ is continuous in $\mathbb{C}_{+}:=\{z \in \mathbb{C} \mid$ $\operatorname{Im}(z) \geq 0\}$ outside the points $z= \pm m$, where it may diverge as $|z \mp m|^{-1 / 2}$. The proof of the proposition relies on a technical result that we now recall.

Lemma 5.1 (Lemma 2.4 of [27]). Let $U \in \mathrm{L}^{q}\left(\mathbb{R}^{2}\right), q \in[1, \infty)$, and assume that $b$ is an admissible magnetic field. Then $p U p \in S_{q}\left[\mathrm{~L}^{2}\left(\mathbb{R}^{2}\right)\right]$, and

$$
\|p U p\|_{S_{q}\left[\mathrm{~L}^{2}\left(\mathbb{R}^{2}\right)\right]}^{q} \leq \frac{b_{0}}{2 \pi} \mathrm{e}^{2 \operatorname{osc}(\widetilde{\varphi})}\|U\|_{\mathrm{L}^{q}\left(\mathbb{R}^{2}\right)}^{q} .
$$


The symbol $y_{+}$denotes the postive part of $y \in \mathbb{R}$.

Proposition 5.2. Let $V$ satisfy Assumption 3.1. Then the operator-valued function

$$
\mathbb{C}_{+} \backslash\{ \pm m\} \ni z \mapsto T_{\operatorname{div}}(z) \in S_{1}(\mathcal{H})
$$

is well-defined and continuous. Moreover, we have for each $\lambda \in \mathbb{R} \backslash\{ \pm m\}$ the bound

$$
\left\|T_{\text {div }}(\lambda)\right\|_{1} \leq \text { Const. }\left(\left|\frac{\lambda+m}{\lambda-m}\right|^{1 / 2}+\left|\frac{\lambda-m}{\lambda+m}\right|^{1 / 2}\right)\left(1+\left(\lambda^{2}-m^{2}\right)_{+}^{1 / 4}\right) .
$$

Proof. We have for each $z \in \mathbb{C} \backslash \sigma\left(H_{0}\right)$ the identity

$$
T_{\text {div }}(z)=M\left(G \otimes J_{z^{2}-m^{2}}\right)\left(\begin{array}{cccc}
(z+m) & 0 & 0 & 0 \\
0 & 0 & 0 & 0 \\
0 & 0 & (z-m) & 0 \\
0 & 0 & 0 & 0
\end{array}\right) M,
$$

where

$$
M:=V^{1 / 2}\left\langle Q_{\perp}\right\rangle^{\nu_{\perp} / 2}\left\langle Q_{3}\right\rangle^{\nu_{3} / 2}, \quad G:=\left\langle Q_{\perp}\right\rangle^{-\nu_{\perp} / 2} p\left\langle Q_{\perp}\right\rangle^{-\nu_{\perp} / 2}, \quad J_{z}:=\left\langle Q_{3}\right\rangle^{-\nu_{3} / 2} R(z)\left\langle Q_{3}\right\rangle^{-\nu_{3} / 2} .
$$

The operator $M$ is bounded due to Assumption 3.1. So

$$
\left\|T_{\text {div }}(z)\right\|_{1} \leq \text { Const. }(|z+m|+|z-m|)\|G\|_{1}\left\|J_{z^{2}-m^{2}}\right\|_{1} .
$$

But we know from Lemma 5.1 that $\|G\|_{1} \leq$ Const., and from [6, Sec. 4.1] that the operator-valued function $\mathbb{C}_{+} \backslash\{0\} \ni z \mapsto J_{z}$ is continuous in the trace norm and admits the bound

$$
\left\|J_{\lambda}\right\|_{1} \leq \text { Const. }\left(1+\lambda_{+}^{1 / 4}\right)|\lambda|^{-1 / 2} \text { for } \lambda \in \mathbb{R} \backslash\{0\} .
$$

It follows that

$$
\left\|T_{\text {div }}(z)\right\|_{1} \leq \text { Const. }\left(\left|\frac{\lambda+m}{\lambda-m}\right|^{1 / 2}+\left|\frac{\lambda-m}{\lambda+m}\right|^{1 / 2}\right)\left(1+\left(\lambda^{2}-m^{2}\right)_{+}^{1 / 4}\right)
$$

for each $\lambda \in \mathbb{R} \backslash\{ \pm m\}$.

In the next proposition, we show that the function $z \mapsto T_{\text {bound }}(z) \in S_{4}(\mathcal{H})$ is continuous in $\mathbb{C} \backslash\left\{\left(-\infty,-\sqrt{m^{2}+\zeta}\right] \cup\right.$ $\left.\left[\sqrt{m^{2}+\zeta}, \infty\right)\right\}$. The symbols $H^{ \pm}$stand for the operators $H^{ \pm}:=H_{\perp}^{ \pm} \otimes 1+1 \otimes P_{3}^{2}$ acting in $\mathrm{L}^{2}\left(\mathbb{R}^{3}\right)$.

Proposition 5.3. Let $V$ satisfy Assumption 3.1. Then the operator-valued function

$$
\mathbb{C} \backslash\left\{\left(-\infty,-\sqrt{m^{2}+\zeta}\right] \cup\left[\sqrt{m^{2}+\zeta}, \infty\right)\right\} \ni z \mapsto T_{\text {bound }}(z) \in S_{4}(\mathcal{H})
$$

is well-defined and continuous. Moreover, we have for each $\lambda \in\left(-\sqrt{m^{2}+\zeta}, \sqrt{m^{2}+\zeta}\right)$ the bound

$$
\left\|T_{\text {bound }}(\lambda)\right\|_{4} \leq \text { Const. }\left(|\lambda|+\lambda^{2}\right)\left(1+\frac{\left(\lambda^{2}-m^{2}+1\right)_{+}}{\zeta+m^{2}-\lambda^{2}}\right)+\text { Const. }
$$

Proof. One has the identity

$$
\left(H_{0}-z\right)^{-1}=H_{0}^{-1}+z\left(1+z H_{0}^{-1}\right)\left(H_{0}^{2}-z^{2}\right)^{-1}
$$

for each $z \in \mathbb{C} \backslash \sigma\left(H_{0}\right)$. Thus the operator $T_{\text {bound }}(z)$ can be written as

$$
\begin{aligned}
& T_{\text {bound }}(z)=M\left(G \otimes S_{z}\right)\left(\begin{array}{cccc}
0 & 0 & 1 & 0 \\
0 & 0 & 0 & 0 \\
1 & 0 & 0 & 0 \\
0 & 0 & 0 & 0
\end{array}\right) M+V^{1 / 2} H_{0}^{-1} \mathrm{P}^{\perp} V^{1 / 2}+z V^{1 / 2}\left(1+z H_{0}^{-1}\right)\left(H_{0}^{2}-z^{2}\right)^{-1} \mathrm{P}^{\perp} V^{1 / 2} \\
& \equiv T_{1}(z)+T_{2}+T_{3}(z)
\end{aligned}
$$


with $M$ and $G$ given in (5.4), and

$$
S_{z}:=\left\langle Q_{3}\right\rangle^{-\nu_{3} / 2} P_{3} R\left(z^{2}-m^{2}\right)\left\langle Q_{3}\right\rangle^{-\nu_{3} / 2} .
$$

The integral kernel of $S_{z}$ is

$$
\frac{i}{2}\left\langle x_{3}\right\rangle^{-\nu_{3} / 2} \frac{\left(x_{3}-x_{3}^{\prime}\right)}{\left|x_{3}-x_{3}^{\prime}\right|} \mathrm{e}^{i \sqrt{z^{2}-m^{2}}\left|x_{3}-x_{3}^{\prime}\right|}\left\langle x_{3}^{\prime}\right\rangle^{-\nu_{3} / 2},
$$

with the branch of $\sqrt{z^{2}-m^{2}}$ chosen so that $\operatorname{Im} \sqrt{z^{2}-m^{2}}>0$. So $S_{z}$ extends to an element of $S_{2}\left[\mathrm{~L}^{2}(\mathbb{R})\right]$ for each $z \in \mathbb{C}$, with $\left\|S_{z}\right\|_{2} \leq$ Const. Since $M$ is bounded and $\|G\|_{1} \leq$ Const., this implies that

$$
\left\|T_{1}(z)\right\|_{2} \leq \text { Const. }\|M\|^{2}\|G\|_{1}\left\|S_{z}\right\|_{2} \leq \text { Const. }
$$

for each $z \in \mathbb{C}$. One also has

$$
\left\|T_{2}\right\|_{4} \leq \text { Const. }
$$

due to (3.3). So, it only remains to bound the term $T_{3}(z)$.

Let $z \in \mathbb{C} \backslash\left\{\left(-\infty,-\sqrt{m^{2}+\zeta}\right] \cup\left[\sqrt{m^{2}+\zeta}, \infty\right)\right\}$ and $P^{\perp}:=1-P$. Then $\left(H^{-}+m^{2}-z^{2}\right)^{-1} P^{\perp}$ and $\left(H^{+}+m^{2}-z^{2}\right)^{-1}$ belong to $\mathscr{B}\left[\mathrm{L}^{2}\left(\mathbb{R}^{3}\right)\right]$, and we have

$$
\left(H^{-}+m^{2}-z^{2}\right)^{-1} P^{\perp}=P^{\perp}\left(H^{-}+m^{2}-z^{2}\right)^{-1} .
$$

Thus

$$
\begin{aligned}
& \left(H_{0}^{2}-z^{2}\right)^{-1} \mathrm{P}^{\perp} V^{1 / 2}=\left(H_{0}^{2}-z^{2}\right)^{-1}\left(\begin{array}{cccc}
P^{\perp} & 0 & 0 & 0 \\
0 & 1 & 0 & 0 \\
0 & 0 & P^{\perp} & 0 \\
0 & 0 & 0 & 1
\end{array}\right) V^{1 / 2} \\
& =\left(\begin{array}{cccc}
P^{\perp}\left(H^{-}+m^{2}-z^{2}\right)^{-1} & 0 & 0 & 0 \\
0 & \left(H^{+}+m^{2}-z^{2}\right)^{-1} & 0 & 0 \\
0 & 0 & P^{\perp}\left(H^{-}+m^{2}-z^{2}\right)^{-1} & 0 \\
0 & 0 & 0 & \left(H^{+}+m^{2}-z^{2}\right)^{-1}
\end{array}\right) V^{1 / 2},
\end{aligned}
$$

and

$$
\left\|\left(H_{0}^{2}-z^{2}\right)^{-1} \mathrm{P}^{\perp} V^{1 / 2}\right\|_{2}^{2} \leq 2\|M\|^{2}\left\{\left\|P^{\perp}\left(H^{-}+m^{2}-z^{2}\right)^{-1} M_{2}\right\|_{2}^{2}+\left\|\left(H^{+}+m^{2}-z^{2}\right)^{-1} M_{2}\right\|_{2}^{2}\right\},
$$

where $M_{2}:=\left\langle Q_{\perp}\right\rangle^{-\nu_{\perp} / 2}\left\langle Q_{3}\right\rangle^{-\nu_{3} / 2}$. But, we know from the proof of [27, Prop. 4.4] that

$$
\left\|P^{\perp}\left(H^{-}+m^{2}-z^{2}\right)^{-1} M_{2}\right\|_{2} \leq \text { Const. } C(z) \quad \text { and } \quad\left\|\left(H^{+}+m^{2}-z^{2}\right)^{-1} M_{2}\right\|_{2} \leq \text { Const. } C(z),
$$

where

$$
C(z):=\sup _{y \in[\zeta, \infty)} \frac{y+1}{\left|y+m^{2}-z^{2}\right|}
$$

It follows that

$$
\left\|T_{3}(z)\right\|_{2} \leq \text { Const. }\left\|z V^{1 / 2}\left(1+z H_{0}^{-1}\right)\right\|\|M\| C(z) \leq \text { Const. }\left(|z|+|z|^{2}\right) C(z) .
$$

The claim follows then by putting together (5.8), (5.9), and (5.10).

In the next lemma we give some results on the imaginary part of the operator $S_{z}$ in $\mathrm{L}^{2}(\mathbb{R})$ appearing in the proof of Proposition 5.3

$$
S_{z}=\left\langle Q_{3}\right\rangle^{-\nu_{3} / 2} P_{3} R\left(z^{2}-m^{2}\right)\left\langle Q_{3}\right\rangle^{-\nu_{3} / 2}, \quad z \in \mathbb{C} \backslash \sigma\left(H_{0}\right), \nu_{3}>1 .
$$

Lemma 5.4. (a) One has $\operatorname{Im} S_{\lambda}=0$ for each $\lambda \in(-m, m)$. 
(b) Let $p \geq 1$ be an integer. Then one has for each $\lambda \in \mathbb{R}$ with $|\lambda|>m$

$$
\left\|\operatorname{lm} S_{\lambda}\right\|_{p} \leq \mathrm{C}_{p},
$$

where $\mathrm{C}_{p}$ is a constant independent of $\lambda$. Furthermore

$$
\lim _{\lambda \rightarrow \pm m,|\lambda|>m}\left\|\operatorname{Im} S_{\lambda}\right\|_{p}=0 .
$$

Proof. (a) This is a direct consequence of the spectral theorem.

(b) Let $\lambda \in \mathbb{R}$ with $|\lambda|>m$. Then one shows by using (5.7) that $\operatorname{Im} S_{\lambda}$ is equal to the rank two operator

$$
\operatorname{Im} S_{\lambda}=\left\langle v_{\lambda}, \cdot\right\rangle u_{\lambda}+\left\langle u_{\lambda}, \cdot\right\rangle v_{\lambda},
$$

with

$$
u_{\lambda}\left(x_{3}\right):=\left\langle x_{3}\right\rangle^{-\nu_{3} / 2} \sin \left(x_{3} \sqrt{\lambda^{2}-m^{2}}\right) \quad \text { and } \quad v_{\lambda}\left(x_{3}\right):=-\frac{i}{2}\left\langle x_{3}\right\rangle^{-\nu_{3} / 2} \cos \left(x_{3} \sqrt{\lambda^{2}-m^{2}}\right) .
$$

Since $\left\langle v_{\lambda}, u_{\lambda}\right\rangle=0$, this implies that

$$
\left|\operatorname{Im} S_{\lambda}\right|^{p}=\left\|u_{\lambda}\right\|^{p}\left\langle v_{\lambda}, \cdot\right\rangle v_{\lambda}+\left\|v_{\lambda}\right\|^{p}\left\langle u_{\lambda}, \cdot\right\rangle u_{\lambda} .
$$

Thus

$$
\left\|\operatorname{Im} S_{\lambda}\right\|_{p}^{p}=\operatorname{Tr}\left(\left|\operatorname{Im} S_{\lambda}\right|^{p}\right)=\left\|u_{\lambda}\right\|^{p}\left\|v_{\lambda}\right\|^{2}+\left\|v_{\lambda}\right\|^{p}\left\|u_{\lambda}\right\|^{2} .
$$

This, together with the equality

$$
\lim _{\lambda \rightarrow \pm m,|\lambda|>m}\left\|u_{\lambda}\right\|=0
$$

implies the claim.

In the next corollary we combine some of the results of Propositions 5.2, 5.3 and Lemma 5.4.

Corollary 5.5. Let $V$ satisfy Assumption 3.1. Then the identity

$$
T(\lambda+i 0)=T_{\text {div }}(\lambda)+T_{\text {bound }}(\lambda)
$$

holds for each $\lambda \in\left(-\sqrt{m^{2}+\zeta}, \sqrt{m^{2}+\zeta}\right) \backslash\{ \pm m\}$, and the estimate

$$
\left\|\operatorname{Im} T_{\text {bound }}(\lambda)\right\|_{p} \leq \text { Const. }\left\|\operatorname{Im} S_{\lambda}\right\|_{p}
$$

holds for each integer $p \geq 1$ an each $\lambda \in\left(-\sqrt{m^{2}+\zeta}, \sqrt{m^{2}+\zeta}\right)$. In particular, we have

$$
\lim _{\lambda \rightarrow \pm m}\left\|\operatorname{Im} T_{\text {bound }}(\lambda)\right\|_{p}=0
$$

due to Lemma 5.4.

Proof. The first identity follows from Propositions 5.2 and 5.3. Let $\lambda \in\left(-\sqrt{m^{2}+\zeta}, \sqrt{m^{2}+\zeta}\right)$. Using (5.6) and the commutation rule (5.3) one obtains that

$$
\operatorname{Im} T_{\text {bound }}(\lambda)=M\left(G \otimes \operatorname{Im} S_{\lambda}\right)\left(\begin{array}{llll}
0 & 0 & 1 & 0 \\
0 & 0 & 0 & 0 \\
1 & 0 & 0 & 0 \\
0 & 0 & 0 & 0
\end{array}\right) M,
$$

with $M$ and $G$ defined in (5.4). Since $M$ is bounded and $\|G\|_{1} \leq$ Const., this implies (5.12). 


\section{Proof of the main results}

We begin this section by showing that the value of $\xi\left(\lambda ; H, H_{ \pm}\right)$as $\lambda \rightarrow \pm m$ is bounded from below and from above by expressions involving only the term $T_{\text {div }}(\lambda)$ of the decomposition $T(\lambda+i 0)=T_{\text {div }}(\lambda)+T_{\text {bound }}(\lambda)$. Then we consider separately the limits $\lambda \rightarrow \pm m$ with $|\lambda|<m$ and the limits $\lambda \rightarrow \pm m$ with $|\lambda|>m$.

We start by recalling two standard properties of the counting functions $n_{ \pm}$. Given two compact operators $T_{1}=T_{1}^{*}$ and $T_{2}=T_{2}^{*}$ in a separable Hilbert space $\mathcal{G}$, we have the Weyl inequalities

$$
n_{ \pm}\left(s_{1}+s_{2} ; T_{1}+T_{2}\right) \leq n_{ \pm}\left(s_{1} ; T_{1}\right)+n_{ \pm}\left(s_{2} ; T_{2}\right) \text { for each } s_{1}, s_{2}>0 .
$$

Moreover, if $T=T^{*}$ belongs to $S_{p}(\mathcal{G})$ for some $p \in[1, \infty)$, then

$$
n_{ \pm}(s ; T) \leq s^{-p}\|T\|_{p}^{p} \quad \text { for each } s>0 .
$$

Proposition 6.1. Let $V$ satisfy Assumption 3.2. Then the estimates

$$
\begin{aligned}
& \int_{\mathbb{R}} \mathrm{d} \mu(t) n_{ \pm}\left(1+\varepsilon ; \operatorname{Re} T_{\operatorname{div}}(\lambda)+t \operatorname{Im} T_{\operatorname{div}}(\lambda)\right)+\mathcal{O}(1) \\
& \leq \mp \xi\left(\lambda ; H_{\mp}, H_{0}\right) \\
& \leq \int_{\mathbb{R}} \mathrm{d} \mu(t) n_{ \pm}\left(1-\varepsilon ; \operatorname{Re} T_{\operatorname{div}}(\lambda)+t \operatorname{Im} T_{\operatorname{div}}(\lambda)\right)+\mathcal{O}(1)
\end{aligned}
$$

hold as $\lambda \rightarrow \pm m$ for each $\varepsilon \in(0,1)$.

Proof. Using (5.11), the Weyl inequalities (6.1), and Lemma 4.1 we get

$$
\begin{aligned}
& \int_{\mathbb{R}} \mathrm{d} \mu(t) n_{ \pm}\left(1+\varepsilon ; \operatorname{Re} T_{\text {div }}(\lambda)+t \operatorname{Im} T_{\operatorname{div}}(\lambda)\right)-n_{\mp}\left(\varepsilon / 2 ; \operatorname{Re} T_{\text {bound }}(\lambda)\right)-\frac{2}{\pi \varepsilon}\left\|\operatorname{Im} T_{\text {bound }}(\lambda)\right\|_{1} \\
& \leq \int_{\mathbb{R}} \mathrm{d} \mu(t) n_{ \pm}(1 ; A(\lambda+i 0)+t B(\lambda+i 0)) \\
& \leq \int_{\mathbb{R}} \mathrm{d} \mu(t) n_{ \pm}\left(1-\varepsilon ; \operatorname{Re} T_{\text {div }}(\lambda)+t \operatorname{Im} T_{\text {div }}(\lambda)\right)+n_{ \pm}\left(\varepsilon / 2 ; \operatorname{Re} T_{\text {bound }}(\lambda)\right)+\frac{2}{\pi \varepsilon}\left\|\operatorname{Im} T_{\text {bound }}(\lambda)\right\|_{1} .
\end{aligned}
$$

Due to (6.2), we have

$$
n_{ \pm}\left(\varepsilon / 2 ; \operatorname{Re} T_{\text {bound }}(\lambda)\right) \leq 16 \varepsilon^{-4}\left\|T_{\text {bound }}(\lambda)\right\|_{4}^{4},
$$

which combined with (5.5) gives

$$
n_{ \pm}\left(\varepsilon / 2 ; \operatorname{Re} T_{\text {bound }}(\lambda)\right)=\mathcal{O}(1) \quad \text { as } \quad \lambda \rightarrow \pm m .
$$

Moreover, we know from (5.13) that

$$
\lim _{\lambda \rightarrow \pm m}\left\|\operatorname{Im} T_{\text {bound }}(\lambda)\right\|_{1}=0 .
$$

So the claim follows from the estimates (6.3) and Formula (4.1)

\subsection{The case $|\lambda|<m$}

In this section we prove asymptotic estimates for $\xi\left(\lambda ; H, H_{ \pm}\right)$as $\lambda \rightarrow \pm m$ with $|\lambda|<m$. We start with a corollary of Proposition 6.1, which follows from the fact that $\operatorname{Im} T_{\operatorname{div}}(\lambda)=0$ and $\operatorname{Re} T_{\operatorname{div}}(\lambda)=T_{\operatorname{div}}(\lambda)$ for $\lambda \in(-m, m)$.

Corollary 6.2. Let $V$ satisfy Assumption 3.2. Then the estimates

$$
n_{ \pm}\left(1+\varepsilon ; T_{\text {div }}(\lambda)\right)+\mathcal{O}(1) \leq \mp \xi\left(\lambda ; H_{\mp}, H_{0}\right) \leq n_{ \pm}\left(1-\varepsilon ; T_{\operatorname{div}}(\lambda)\right)+\mathcal{O}(1)
$$

hold as $\lambda \rightarrow \pm m,|\lambda|<m$, for each $\varepsilon \in(0,1)$. 
Define the bounded operators $K_{ \pm}: \mathcal{H} \rightarrow \mathrm{L}^{2}\left(\mathbb{R}^{2} ; \mathbb{C}^{4}\right)$ by

$$
\begin{aligned}
& \left(K_{+} \varphi\right)\left(x_{\perp}\right):=\int_{\mathbb{R}^{3}} \mathrm{~d} x_{\perp}^{\prime} \mathrm{d} x_{3}^{\prime} p\left(x_{\perp}, x_{\perp}^{\prime}\right)\left(\begin{array}{cccc}
1 & 0 & 0 & 0 \\
0 & 0 & 0 & 0 \\
0 & 0 & 0 & 0 \\
0 & 0 & 0 & 0
\end{array}\right) V^{1 / 2}\left(x_{\perp}^{\prime}, x_{3}^{\prime}\right) \varphi\left(x_{\perp}^{\prime}, x_{3}^{\prime}\right), \\
& \left(K_{-} \varphi\right)\left(x_{\perp}\right):=\int_{\mathbb{R}^{3}} \mathrm{~d} x_{\perp}^{\prime} \mathrm{d} x_{3}^{\prime} p\left(x_{\perp}, x_{\perp}^{\prime}\right)\left(\begin{array}{cccc}
0 & 0 & 0 & 0 \\
0 & 0 & 0 & 0 \\
0 & 0 & 1 & 0 \\
0 & 0 & 0 & 0
\end{array}\right) V^{1 / 2}\left(x_{\perp}^{\prime}, x_{3}^{\prime}\right) \varphi\left(x_{\perp}^{\prime}, x_{3}^{\prime}\right),
\end{aligned}
$$

where $p(\cdot, \cdot)$ is the integral kernel of the projection $p$. One shows easily that $K_{ \pm}^{*}: \mathrm{L}^{2}\left(\mathbb{R}^{2} ; \mathbb{C}^{4}\right) \rightarrow \mathcal{H}$ are given by

$$
\begin{aligned}
& \left(K_{+}^{*} \psi\right)\left(x_{\perp}, x_{3}\right)=V^{1 / 2}\left(x_{\perp}, x_{3}\right)\left(\begin{array}{cccc}
1 & 0 & 0 & 0 \\
0 & 0 & 0 & 0 \\
0 & 0 & 0 & 0 \\
0 & 0 & 0 & 0
\end{array}\right)(p \psi)\left(x_{\perp}\right), \\
& \left(K_{-}^{*} \psi\right)\left(x_{\perp}, x_{3}\right)=V^{1 / 2}\left(x_{\perp}, x_{3}\right)\left(\begin{array}{cccc}
0 & 0 & 0 & 0 \\
0 & 0 & 0 & 0 \\
0 & 0 & 1 & 0 \\
0 & 0 & 0 & 0
\end{array}\right)(p \psi)\left(x_{\perp}\right),
\end{aligned}
$$

and that

$$
O_{+}(\lambda):=\frac{1}{2}\left(\frac{m+\lambda}{m-\lambda}\right)^{1 / 2} K_{+}^{*} K_{+} \quad \text { and } \quad O_{-}(\lambda):=-\frac{1}{2}\left(\frac{m-\lambda}{m+\lambda}\right)^{1 / 2} K_{-}^{*} K_{-}
$$

belong to $S_{2}(\mathcal{H})$ for each $\lambda \in(-m, m)$.

In the next proposition we show that the functions $n_{ \pm}\left(\cdot ; T_{\operatorname{div}}(\lambda)\right)$ as $\lambda \rightarrow \pm m,|\lambda|<m$, can be bounded, up to $\mathcal{O}(1)$ terms, from below and from above by expressions involving $O_{ \pm}(\lambda)$.

Proposition 6.3. Let $V$ satisfy Assumption 3.2. Then the estimates

$$
\begin{aligned}
n_{+}\left((1+\varepsilon) s ; O_{+}(\lambda)\right)+\mathcal{O}(1) & \leq n_{+}\left(s ; T_{\operatorname{div}}(\lambda)\right) \leq n_{+}\left((1-\varepsilon) s ; O_{+}(\lambda)\right)+\mathcal{O}(1), \\
\mathcal{O}(1) & \leq n_{-}\left(s ; T_{\operatorname{div}}(\lambda)\right) \leq \mathcal{O}(1),
\end{aligned}
$$

hold as $\lambda \nearrow m$, for each $\varepsilon \in(0,1)$ and $s>0$, and the estimates

$$
\begin{aligned}
\mathcal{O}(1) & \leq n_{+}\left(s ; T_{\operatorname{div}}(\lambda)\right) \leq \mathcal{O}(1), \\
n_{-}\left((1+\varepsilon) s ; O_{-}(\lambda)\right)+\mathcal{O}(1) & \leq n_{-}\left(s ; T_{\operatorname{div}}(\lambda)\right) \leq n_{-}\left((1-\varepsilon) s ; O_{-}(\lambda)\right)+\mathcal{O}(1),
\end{aligned}
$$

hold as $\lambda \searrow-m$, for each $\varepsilon \in(0,1)$ and $s>0$.

Proof. We only give the proof of (6.4)-(6.5), since the proof of (6.6)-(6.7) is similar. In point (i) below we show that the difference $T_{\operatorname{div}}(\lambda)-O_{+}(\lambda)$ can be approximated in norm, as $\lambda \nearrow m$, by a compact operator independent of $\lambda$. Then we prove (6.4)-(6.5) in point (ii) by using this result.

(i) Let $\lambda \in(-m, m)$ and take $\nu^{\prime} \in(3, \nu)$. A direct calculation shows that

$$
T_{\operatorname{div}}(\lambda)-O_{+}(\lambda)=\widetilde{M}\left(G_{\nu-\nu^{\prime}} \otimes J_{\nu^{\prime}}^{(\lambda)}\right)\left(\begin{array}{cccc}
(\lambda+m) & 0 & 0 & 0 \\
0 & 0 & 0 & 0 \\
0 & 0 & (\lambda-m) & 0 \\
0 & 0 & 0 & 0
\end{array}\right) \widetilde{M}+O_{-}(\lambda)
$$

where $J_{\nu^{\prime}}^{(\lambda)}: \mathrm{L}^{2}(\mathbb{R}) \rightarrow \mathrm{L}^{2}(\mathbb{R})$ is given by

$$
\left(J_{\nu^{\prime}}^{(\lambda)} \psi\right)\left(x_{3}\right):=-\left\langle x_{3}\right\rangle^{-\nu^{\prime} / 2} \int_{\mathbb{R}} \mathrm{d} x_{3}^{\prime} \frac{\mathrm{e}^{-\frac{1}{2} \sqrt{m^{2}-\lambda^{2}}\left|x_{3}-x_{3}^{\prime}\right|}}{\sqrt{m^{2}-\lambda^{2}}} \sinh \left(\frac{\sqrt{m^{2}-\lambda^{2}}\left|x_{3}-x_{3}^{\prime}\right|}{2}\right)\left\langle x_{3}^{\prime}\right\rangle^{-\nu^{\prime} / 2} \psi\left(x_{3}^{\prime}\right)
$$

and

$$
\begin{aligned}
\widetilde{M} & :=V^{1 / 2}\left\langle Q_{\perp}\right\rangle^{\left(\nu-\nu^{\prime}\right) / 2}\left\langle Q_{3}\right\rangle^{\nu^{\prime} / 2} \\
G_{\nu-\nu^{\prime}} & :=\left\langle Q_{\perp}\right\rangle^{-\left(\nu-\nu^{\prime}\right) / 2} p\left\langle Q_{\perp}\right\rangle^{-\left(\nu-\nu^{\prime}\right) / 2} .
\end{aligned}
$$

The operator $\widetilde{M}$ is bounded due to Assumption $3.2, G_{\nu-\nu^{\prime}}$ is compact in $\mathrm{L}^{2}\left(\mathbb{R}^{2} ; \mathbb{C}^{4}\right)$ due to Lemma 5.1 , and $O_{-}(\lambda)$ satisfies

$$
\lim _{\lambda \rightarrow m,|\lambda|<m}\left\|O_{-}(\lambda)\right\|_{2}=0
$$


Define

$$
T_{ \pm}:=\widetilde{M}\left(G_{\nu-\nu^{\prime}} \otimes J_{\nu^{\prime}}^{(m)}\right)\left(\begin{array}{cccc}
(m \pm m) & 0 & 0 & 0 \\
0 & 0 & 0 & 0 \\
0 & 0 & -(m \mp m) & 0 \\
0 & 0 & 0 & 0
\end{array}\right) \widetilde{M}
$$

with $J_{\nu^{\prime}}^{(m)}: \mathrm{L}^{2}(\mathbb{R}) \rightarrow \mathrm{L}^{2}(\mathbb{R})$ given by

$$
\left(J_{\nu^{\prime}}^{(m)} \psi\right)\left(x_{3}\right):=-\frac{1}{2}\left\langle x_{3}\right\rangle^{-\nu^{\prime} / 2} \int_{\mathbb{R}} \mathrm{d} x_{3}^{\prime}\left|x_{3}-x_{3}^{\prime}\right|\left\langle x_{3}^{\prime}\right\rangle^{-\nu^{\prime} / 2} \psi\left(x_{3}^{\prime}\right) .
$$

Since $\nu^{\prime}>3, J_{\nu^{\prime}}^{(m)}$ belongs to $S_{2}\left[\mathrm{~L}^{2}(\mathbb{R})\right]$, and $T_{ \pm}$is compact in $\mathcal{H}$. Moreover, by using Lebesgue's dominated convergence theorem, one shows that

$$
\lim _{\lambda \rightarrow \pm m,|\lambda|<m}\left\|J_{\nu^{\prime}}^{(m)}-J_{\nu^{\prime}}^{(\lambda)}\right\|_{2}^{2}=0
$$

This, together with (6.8), (6.11) and (6.12), implies that

$$
\lim _{\lambda \nearrow m}\left\|T_{\operatorname{div}}(\lambda)-O_{+}(\lambda)-T_{+}\right\|=0 .
$$

(ii) Take $\lambda \in(-m, m), \varepsilon \in(0,1)$, and $s>0$. Using the Weyl inequalities (6.1) we get

$$
\begin{aligned}
n_{ \pm}\left((1+\varepsilon) s ; O_{+}(\lambda)\right)-n_{\mp}\left(\varepsilon s ; T_{\operatorname{div}}(\lambda)-O_{+}(\lambda)\right) & \leq n_{ \pm}\left(s ; T_{\operatorname{div}}(\lambda)\right) \\
& \leq n_{ \pm}\left((1-\varepsilon) s ; O_{+}(\lambda)\right)+n_{ \pm}\left(\varepsilon s ; T_{\operatorname{div}}(\lambda)-O_{+}(\lambda)\right)
\end{aligned}
$$

Now we have $n_{-}\left(t ; O_{+}(\lambda)\right)=0$ for each $t>0$ and $\lambda \in(-m, m)$, since $O_{+}(\lambda)$ is a positive operator. So, to prove (6.4)-(6.5), it is sufficient to show that $n_{ \pm}\left(\varepsilon s ; T_{\operatorname{div}}(\lambda)-O_{+}(\lambda)\right)=\mathcal{O}(1)$ as $\lambda \nearrow m$, for each $\varepsilon \in(0,1)$ and $s>0$. Let $t>0$ be fixed. Then we know from (6.13) that we can chose $\lambda_{+} \in(-m, m)$, close enough to $m$, so that $\left\|T_{\operatorname{div}}\left(\lambda_{+}\right)-O_{+}\left(\lambda_{+}\right)-T_{+}\right\|<t / 2$. Thus, using again the Weyl inequalities, we get

$$
n_{ \pm}\left(t ; T_{\operatorname{div}}\left(\lambda_{+}\right)-O_{+}\left(\lambda_{+}\right)\right) \leq n_{ \pm}\left(t / 2 ; T_{\operatorname{div}}\left(\lambda_{+}\right)-O_{+}\left(\lambda_{+}\right)-T_{+}\right)+n_{ \pm}\left(t / 2 ; T_{+}\right)=n_{ \pm}\left(t / 2 ; T_{+}\right) .
$$

Since the r.h.s. is independent of $\lambda_{+}$we have shown that $n_{ \pm}\left(t ; T_{\operatorname{div}}(\lambda)-O_{+}(\lambda)\right)=\mathcal{O}(1)$ as $\lambda \nearrow m$. This concludes the proof of (6.4)-(6.5).

We show now that the counting functions $n_{ \pm}\left(\cdot ; O_{ \pm}(\lambda)\right)$ in Proposition 6.3 can be rewritten in terms of Berezin-Toeplitz type operators. Define for each $\lambda \in(-m, m)$

$$
\omega_{+}(\lambda):=\frac{1}{2}\left(\frac{m+\lambda}{m-\lambda}\right)^{1 / 2} p W_{+} p \quad \text { and } \quad \omega_{-}(\lambda):=-\frac{1}{2}\left(\frac{m-\lambda}{m+\lambda}\right)^{1 / 2} p W_{-} p
$$

where the functions $W_{ \pm}: \mathbb{R}^{2} \rightarrow \mathbb{R}$ are given by

$$
W_{+}\left(x_{\perp}\right):=\int_{\mathbb{R}} \mathrm{d} x_{3} V_{11}\left(x_{\perp}, x_{3}\right) \quad \text { and } \quad W_{-}\left(x_{\perp}\right):=\int_{\mathbb{R}} \mathrm{d} x_{3} V_{33}\left(x_{\perp}, x_{3}\right) .
$$

Under the condition (3.5) one has

$$
0 \leq W_{ \pm}\left(x_{\perp}\right) \leq \text { Const. }\left\langle x_{\perp}\right\rangle^{-\nu+1} \text { for all } x_{\perp} \in \mathbb{R}^{2}
$$

and $\omega_{ \pm}(\lambda) \in S_{1}\left[\mathrm{~L}^{2}\left(\mathbb{R}^{2}\right)\right]$ if $V$ satisfies Assumption 3.1 (see Lemma 5.1). Moreover, one has the following.

Proposition 6.4. Let $V$ satisfy Assumption 3.1. Then we have for each $\lambda \in(-m, m)$ and $s>0$

$$
n_{ \pm}\left(s ; O_{ \pm}(\lambda)\right)=n_{ \pm}\left(s ; \omega_{ \pm}(\lambda)\right) .
$$


Proof. Given $s>0$ and two separable Hilbert spaces $\mathcal{H}_{1}, \mathcal{H}_{2}$, one has

$$
n_{ \pm}\left(s ; B^{*} B\right)=n_{ \pm}\left(s ; B B^{*}\right)
$$

for any $B \in \mathscr{B}\left(\mathcal{H}_{1}, \mathcal{H}_{2}\right)$ such that $B^{*} B \in S_{\infty}\left(\mathcal{H}_{1}\right)$. Moreover, one can easily check that

$$
K_{+} K_{+}^{*}=\left(\begin{array}{llll}
1 & 0 & 0 & 0 \\
0 & 0 & 0 & 0 \\
0 & 0 & 0 & 0 \\
0 & 0 & 0 & 0
\end{array}\right) p W_{+} p \quad \text { and } \quad K_{-} K_{-}^{*}=\left(\begin{array}{cccc}
0 & 0 & 0 & 0 \\
0 & 0 & 0 & 0 \\
0 & 0 & 1 & 0 \\
0 & 0 & 0 & 0
\end{array}\right) p W_{-} p
$$

Thus

$$
n_{+}\left(s ; O_{+}(\lambda)\right)=n_{+}\left(s ; \frac{1}{2}\left(\frac{m+\lambda}{m-\lambda}\right)^{1 / 2}\left(\begin{array}{cccc}
1 & 0 & 0 & 0 \\
0 & 0 & 0 & 0 \\
0 & 0 & 0 & 0 \\
0 & 0 & 0 & 0
\end{array}\right) p W_{+} p\right)=n_{+}\left(s ; \frac{1}{2}\left(\frac{m+\lambda}{m-\lambda}\right)^{1 / 2} p W_{+} p\right)=n_{+}\left(s ; \omega_{+}(\lambda)\right) .
$$

The proof of the second equality in (6.15) is similar.

The next theorem is direct consequence of Corollary 6.2 and Propositions 6.3-6.4.

Theorem 6.5. Let $V$ satisfy Assumption 3.2. Then one has for each $\varepsilon \in(0,1)$

$$
\begin{aligned}
\mathcal{O}(1) & \leq \xi\left(\lambda ; H_{+}, H_{0}\right) \leq \mathcal{O}(1) \\
-n_{+}\left(1-\varepsilon ; \omega_{+}(\lambda)\right)+\mathcal{O}(1) & \leq \xi\left(\lambda ; H_{-}, H_{0}\right) \leq-n_{+}\left(1+\varepsilon ; \omega_{+}(\lambda)\right)+\mathcal{O}(1)
\end{aligned}
$$

as $\lambda \nearrow m$, and

$$
\begin{aligned}
n_{-}\left(1+\varepsilon ; \omega_{-}(\lambda)\right)+\mathcal{O}(1) & \leq \xi\left(\lambda ; H_{+}, H_{0}\right) \leq n_{-}\left(1-\varepsilon ; \omega_{-}(\lambda)\right)+\mathcal{O}(1) \\
\mathcal{O}(1) & \leq \xi\left(\lambda ; H_{-}, H_{0}\right) \leq \mathcal{O}(1)
\end{aligned}
$$

as $\lambda \searrow-m$.

Remark 6.6. The inequalities (6.17) together with Remark 4.5 imply that the eigenvalues of $H_{0}+V$ in $(-m, m)$ near $+m$ (if any) do not accumulate at $+m$. On the other hand the inequalities (6.18) tell us that the number of eigenvalues of $H_{0}-V$ in $(-m, m)$ near $\lambda=+m$ scales, up to $\mathcal{O}(1)$ terms, as

$$
n_{+}\left(s ; \omega_{+}(\lambda)\right) \equiv \operatorname{rank} E^{p W_{+} p}\left(\left(s\left(\frac{m-\lambda}{m+\lambda}\right)^{1 / 2}, \infty\right)\right)
$$

with $s \approx 2$. Accordingly, the problem of counting the number of eigenvalues of $H_{0}-V$ in $(-m, m)$ near $+m$ reduces to the problem of counting the number of eigenvalues of the positive Berezin-Toeplitz type operator $p W_{+}$p near 0 . The inequalities (6.19)-(6.20) lead to similar conclusions on the number of eigenvalues of $H_{0} \pm V$ in $(-m, m)$ near $-m$.

One can compare these results with the results of [8] and [19] on the finiteness in $(-m, m)$ of the discrete spectrum of the Dirac operator perturbed by a matrix potential $Q \equiv\left\{Q_{j k}(x)\right\}_{j, k=1}^{4}$. In Corollary 2.2 of [8], the author shows that the spectrum in $(-m, m)$ of the Dirac operator perturbed by $Q$ is finite if the $2 \times 2$ diagonal blocks of $Q$ are of order $\mathcal{O}\left(|x|^{-2-\delta}\right)$ and the anti-diagonal blocks are of order $\mathcal{O}\left(|x|^{-1-\delta}\right)$, for some $\delta>0$ as $|x| \rightarrow \infty$. In Corollary 2.1 of [19], the authors show that the Dirac operator perturbed by $\gamma Q$, with $|\gamma|$ small enough and

$$
\left|Q_{j k}(x)\right| \leq\langle x\rangle^{-2}, \quad j, k \in\{1, \ldots, 4\},
$$

does not have any point spectrum. Therefore, in our case where $Q=-\alpha_{1} a_{1}-\alpha_{2} a_{2}+V$, we would not have had any accumulation of eigenvalues in $(-m, m)$ if we would have imposed such decay assumptions on the magnetic part $-\alpha_{1} a_{1}-\alpha_{2} a_{2}$ of the perturbation.

As seen in Theorem 6.5 the behaviour of the function $\xi\left(\cdot ; H_{ \pm}, H_{0}\right)$ in $(-m, m)$ depends on the distribution of eigenvalues of the trace class operator $p W_{\mp} p$. In our next proposition we shall exhibit different types of behaviours depending on the choice of the functions $V_{11}$ and $V_{33}$ appearing in $W_{ \pm}$. For that purpose, we first have to recall some technical results taken from [27], [29] and [30]. 
In the first lemma, an integrated density of states (IDS) for the operator $H_{\perp}^{-}$in $\mathrm{L}^{2}\left(\mathbb{R}^{2}\right)$ is defined as follows (see e.g. [10,18]): Let $\chi_{r, x_{\perp}}$ be the characteristic function of the square $x_{\perp}+(-r / 2, r / 2)^{2}$, with $x_{\perp} \in \mathbb{R}^{2}$ and $r>0$. Then a non-increasing function $\varrho:[0, \infty) \rightarrow \mathbb{R}$ is called IDS for the operator $H_{\perp}^{-}$if for each $x_{\perp} \in \mathbb{R}^{2}$ it satisfies

$$
\varrho(\lambda)=\lim _{r \rightarrow \infty} r^{-2} \operatorname{Tr}\left[\chi_{r, x_{\perp}}\left(Q_{\perp}\right) E^{H_{\perp}^{-}}((-\infty, \lambda)) \chi_{r, x_{\perp}}\left(Q_{\perp}\right)\right]
$$

for each point $\lambda \in \mathbb{R}$ of continuity of $\varrho$.

Lemma 6.7 (Lemma 3.3 of [27]). Let $U \in C^{1}\left(\mathbb{R}^{2}\right)$ satisfy

$$
0 \leq U\left(x_{\perp}\right) \leq \text { Const. }\left\langle x_{\perp}\right\rangle^{-\alpha} \quad \text { and } \quad\left|(\nabla U)\left(x_{\perp}\right)\right| \leq \text { Const. }\left\langle x_{\perp}\right\rangle^{-\alpha-1}
$$

for all $x \in \mathbb{R}^{2}$ and some $\alpha>0$. Assume moreover that

- $U\left(x_{\perp}\right)=u\left(\frac{x_{\perp}}{\left|x_{\perp}\right|}\right)(1+o(1))$ as $\left|x_{\perp}\right| \rightarrow \infty$, for some nonzero $u \in C\left(\mathbb{S}^{1}\right)$,

- $b$ is an admissible magnetic field,

- there exists an IDS $\varrho_{b}$ for the operator $H_{\perp}^{-}$.

Then we have

$$
n_{+}(s ; p U p)=\frac{b_{0}}{2 \pi}\left|\left\{x_{\perp} \in \mathbb{R}^{2} \mid U\left(x_{\perp}\right)>s\right\}\right|(1+o(1))=\Psi_{\alpha}\left(s ; u, b_{0}\right)(1+o(1)) \quad \text { as } \quad s \searrow 0,
$$

where $|\cdot|$ denotes the Lebesgue measure, and

$$
\Psi_{\alpha}\left(s ; u, b_{0}\right):=\frac{s^{-2 / \alpha} b_{0}}{4 \pi} \int_{\mathbb{S}^{1}} \mathrm{~d} \vartheta u(\vartheta)^{2 / \alpha}, \quad s>0 .
$$

Lemma 6.8 (Lemma 3.4 of [27]). Let $0 \leq U \in \mathrm{L}^{\infty}\left(\mathbb{R}^{2}\right)$. Assume that

$$
\ln \left(U\left(x_{\perp}\right)\right)=-\eta\left|x_{\perp}\right|^{2 \beta}(1+o(1)) \text { as }\left|x_{\perp}\right| \rightarrow \infty,
$$

for some $\eta, \beta>0$. Let $b$ be an admissible magnetic field. Then we have

$$
n_{+}(s ; p U p)=\Phi_{\beta}\left(s, \eta, b_{0}\right)(1+o(1)) \quad \text { as } s \searrow 0
$$

where

$$
\Phi_{\beta}\left(s, \eta, b_{0}\right):=\left\{\begin{array}{ll}
\frac{b_{0}}{2 \eta^{1 / \beta}}|\ln (s)|^{1 / \beta} & \text { if } \beta \in(0,1), \\
\frac{1}{\ln \left(1+2 \eta / b_{0}\right)}|\ln (s)| & \text { if } \beta=1, \\
\frac{\beta}{\beta-1}(\ln |\ln (s)|)^{-1}|\ln (s)| & \text { if } \beta>1,
\end{array} \quad s \in\left(0, \mathrm{e}^{-1}\right) .\right.
$$

Lemma 6.9 (Lemma 3.5 of [27]). Let $0 \leq U \in \mathrm{L}^{\infty}\left(\mathbb{R}^{2}\right)$. Assume that the support of $U$ is compact, and that there exists a constant $\mathrm{C}>0$ such that $U \geq \mathrm{C}$ on an open non-empty subset of $\mathbb{R}^{2}$. Let b be an admissible magnetic field. Then we have

$$
n_{+}(s ; p U p)=\Phi_{\infty}(s)(1+o(1)) \quad \text { as } \quad s \searrow 0,
$$

where

$$
\Phi_{\infty}(s):=(\ln |\ln (s)|)^{-1}|\ln (s)|, \quad s \in\left(0, \mathrm{e}^{-1}\right) .
$$

Combining Theorem 6.5 with Lemmas 6.7-6.9 we obtain the behaviour of $\xi\left(\lambda ; H_{ \pm}, H_{0}\right)$ as $|\lambda| \rightarrow m$, $|\lambda|<m$, when the functions $W_{ \pm}$admit a power-like or exponential decay at infinity, or when they have a compact support.

Proposition 6.10. Let $V$ satisfy Assumption 3.2. 
(a) Assume that the hypotheses of Lemma 6.7 hold with $U_{ \pm}=W_{ \pm}$and $\alpha=\nu-1$. Then we have

$$
\xi\left(\lambda ; H_{-}, H_{0}\right)=-\Psi_{\nu-1}\left(2\left(\frac{m-\lambda}{m+\lambda}\right)^{1 / 2} ; u_{+}, b_{0}\right)(1+o(1)) \quad \text { as } \quad \lambda \nearrow m,
$$

and

$$
\xi\left(\lambda ; H_{+}, H_{0}\right)=\Psi_{\nu-1}\left(2\left(\frac{m+\lambda}{m-\lambda}\right)^{1 / 2} ; u_{-}, b_{0}\right)(1+o(1)) \quad \text { as } \quad \lambda \searrow-m,
$$

with $\Psi_{\nu-1}$ given by Equation (6.21).

(b) Assume that the hypotheses of Lemma 6.8 hold with $U_{ \pm}=W_{ \pm}$. Then we have

$$
\xi\left(\lambda ; H_{-}, H_{0}\right)=-\Phi_{\beta_{+}}\left(2\left(\frac{m-\lambda}{m+\lambda}\right)^{1 / 2} ; \eta_{+}, b_{0}\right)(1+o(1)) \text { as } \lambda \nearrow m
$$

and

$$
\xi\left(\lambda ; H_{+}, H_{0}\right)=\Phi_{\beta_{-}}\left(2\left(\frac{m+\lambda}{m-\lambda}\right)^{1 / 2} ; \eta_{-}, b_{0}\right)(1+o(1)) \text { as } \quad \lambda \searrow-m,
$$

with $\beta_{ \pm} \in(0, \infty)$ and $\Phi_{\beta_{ \pm}}$given by Equation (6.22).

(c) Assume that the hypotheses of Lemma 6.9 hold with $U_{ \pm}=W_{ \pm}$. Then we have

$$
\xi\left(\lambda ; H_{-}, H_{0}\right)=-\Phi_{\infty}\left(2\left(\frac{m-\lambda}{m+\lambda}\right)^{1 / 2}\right)(1+o(1)) \text { as } \lambda \nearrow m,
$$

and

$$
\xi\left(\lambda ; H_{+}, H_{0}\right)=\Phi_{\infty}\left(2\left(\frac{m+\lambda}{m-\lambda}\right)^{1 / 2}\right)(1+o(1)) \quad \text { as } \quad \lambda \searrow-m,
$$

with $\Phi_{\infty}$ given by Equation (6.23).

The estimates of Proposition 6.10 are consistent with the ones of [27, Cor. 3.6], where the corresponding situation for magnetic Pauli operators is considered.

\subsection{The case $|\lambda|>m$}

In this section we prove asymptotic estimates for $\xi\left(\lambda ; H, H_{ \pm}\right)$as $\lambda \rightarrow \pm m$, when $|\lambda|>m$. We start by showing an estimate for $n_{ \pm}\left(s ; \operatorname{Re} T_{\operatorname{div}}(\lambda)\right)$.

Proposition 6.11. Let $V$ satisfy Assumption 3.2. Then the estimates

$$
n_{ \pm}\left(s ; \operatorname{Re} T_{\operatorname{div}}(\lambda)\right)=\mathcal{O}(1) \quad \text { as } \quad \lambda \rightarrow \pm m,|\lambda|>m
$$

hold for each $s>0$.

Proof. Take $\lambda \in \mathbb{R}$ with $|\lambda|>m$, and let $\nu^{\prime} \in(3, \nu)$. Then we have

$$
\operatorname{Re} T_{\text {div }}(\lambda)=\widetilde{M}\left(G_{\nu-\nu^{\prime}} \otimes R_{\nu^{\prime}}^{(\lambda)}\right)\left(\begin{array}{cccc}
(\lambda+m) & 0 & 0 & 0 \\
0 & 0 & 0 & 0 \\
0 & 0 & (\lambda-m) & 0 \\
0 & 0 & 0 & 0
\end{array}\right) \widetilde{M}
$$

with $\widetilde{M}$ and $G_{\nu-\nu^{\prime}}$ as in (6.9)-(6.10), and

$$
R_{\nu^{\prime}}^{(\lambda)}:=\left\langle Q_{3}\right\rangle^{-\nu^{\prime} / 2} \operatorname{Re} R\left(\lambda^{2}-m^{2}\right)\left\langle Q_{3}\right\rangle^{-\nu^{\prime} / 2}
$$

By using Lebesgue's dominated convergence theorem, one shows that

$$
\lim _{\lambda \rightarrow \pm m,|\lambda|>m}\left\|\operatorname{Re} T_{\operatorname{div}}(\lambda)-T_{ \pm}\right\|=0
$$

with $T_{ \pm}$as in (6.12). So the claim can be proved as in point (ii) of the proof of Proposition 6.3. 
The next result follows from applying Propositions 6.1 and 6.11, the Weyl inequalities (6.1) and the identities [12, Sec. 5.4]

$$
\int_{\mathbb{R}} \mathrm{d} \mu(t) n_{ \pm}(s ; t T)=\pi^{-1} \operatorname{Tr} \arctan \left(s^{-1} T\right), \quad s>0,
$$

where $T \in S_{1}(\mathcal{H}), T=T^{*} \geq 0$. We also use the fact that $\operatorname{sgn}(\lambda) \operatorname{Im} T_{\text {div }}(\lambda)$ is a positive operator if $|\lambda|>m$.

Corollary 6.12. Let $V$ satisfy Assumption 3.2. Then the estimates

$$
\begin{aligned}
& \pi^{-1} \operatorname{Tr} \arctan \left[(1+\varepsilon)^{-1} \operatorname{sgn}(\lambda) \operatorname{Im} T_{\text {div }}(\lambda)\right]+\mathcal{O}(1) \\
& \leq \mp \xi\left(\lambda ; H_{\mp}, H_{0}\right) \\
& \leq \pi^{-1} \operatorname{Tr} \arctan \left[(1-\varepsilon)^{-1} \operatorname{sgn}(\lambda) \operatorname{Im} T_{\text {div }}(\lambda)\right]+\mathcal{O}(1)
\end{aligned}
$$

hold as $\lambda \rightarrow \pm m,|\lambda|>m$, for each $\varepsilon \in(0,1)$.

As in the case $|\lambda|<m$, we introduce auxiliary operators in order to express the lower and upper bounds for $\mp \xi\left(\lambda ; H_{\mp}, H_{0}\right)$ in terms of Berezin-Toeplitz type operators. For $\lambda \in \mathbb{R}$ with $|\lambda|>m$, we define the operators $K_{1, \lambda}, K_{2, \lambda}: \mathcal{H} \rightarrow \mathrm{L}^{2}\left(\mathbb{R}^{2} ; \mathbb{C}^{4}\right)$ by

$$
\begin{aligned}
& \left(K_{1, \lambda} \varphi\right)\left(x_{\perp}\right):=\int_{\mathbb{R}^{3}} \mathrm{~d} x_{\perp}^{\prime} \mathrm{d} x_{3}^{\prime} p\left(x_{\perp}, x_{\perp}^{\prime}\right) \cos \left(x_{3}^{\prime} \sqrt{\lambda^{2}-m^{2}}\right)\left(\begin{array}{cccc}
\sqrt{|\lambda+m|} & 0 & 0 & 0 \\
0 & 0 & 0 & 0 \\
0 & 0 & \sqrt{|\lambda-m|} \\
0 & 0 & 0 & 0
\end{array}\right) V^{1 / 2}\left(x_{\perp}^{\prime}, x_{3}^{\prime}\right) \varphi\left(x_{\perp}^{\prime}, x_{3}^{\prime}\right), \\
& \left(K_{2, \lambda} \varphi\right)\left(x_{\perp}\right):=\int_{\mathbb{R}^{3}} \mathrm{~d} x_{\perp}^{\prime} \mathrm{d} x_{3}^{\prime} p\left(x_{\perp}, x_{\perp}^{\prime}\right) \sin \left(x_{3}^{\prime} \sqrt{\lambda^{2}-m^{2}}\right)\left(\begin{array}{cccc}
\sqrt{|\lambda+m|} & 0 & 0 & 0 \\
0 & 0 & 0 & 0 \\
0 & 0 & \sqrt{|\lambda-m|} & 0 \\
0 & 0 & 0 & 0
\end{array}\right) V^{1 / 2}\left(x_{\perp}^{\prime}, x_{3}^{\prime}\right) \varphi\left(x_{\perp}^{\prime}, x_{3}^{\prime}\right) .
\end{aligned}
$$

Direct calculations show that the adjoint operators $K_{1, \lambda}^{*}, K_{2, \lambda}^{*}: \mathrm{L}^{2}\left(\mathbb{R}^{2} ; \mathbb{C}^{4}\right) \rightarrow \mathcal{H}$ are given by

$$
\begin{aligned}
& \left(K_{1, \lambda}^{*} \psi\right)\left(x_{\perp}, x_{3}\right)=\cos \left(x_{3} \sqrt{\lambda^{2}-m^{2}}\right) V^{1 / 2}\left(x_{\perp}, x_{3}\right)\left(\begin{array}{cccc}
\sqrt{|\lambda+m|} & 0 & 0 & 0 \\
0 & 0 & 0 & 0 \\
0 & 0 & \sqrt{|\lambda-m|} \\
0 & 0 & 0 & 0
\end{array}\right)(p \psi)\left(x_{\perp}\right), \\
& \left(K_{2, \lambda}^{*} \psi\right)\left(x_{\perp}, x_{3}\right)=\sin \left(x_{3} \sqrt{\lambda^{2}-m^{2}}\right) V^{1 / 2}\left(x_{\perp}, x_{3}\right)\left(\begin{array}{cccc}
\sqrt{|\lambda+m|} & 0 & 0 & 0 \\
0 & 0 & 0 & 0 \\
0 & 0 & \sqrt{|\lambda-m|} & 0 \\
0 & 0 & 0 & 0
\end{array}\right)(p \psi)\left(x_{\perp}\right),
\end{aligned}
$$

and that

$$
\operatorname{sgn}(\lambda) \operatorname{Im} T_{\text {div }}(\lambda)=\frac{1}{2 \sqrt{\lambda^{2}-m^{2}}}\left(K_{1, \lambda}^{*} K_{1, \lambda}+K_{2, \lambda}^{*} K_{2, \lambda}\right) .
$$

This last equation can be written more compactly as

$$
\operatorname{sgn}(\lambda) \operatorname{Im} T_{\operatorname{div}}(\lambda)=\frac{1}{2 \sqrt{\lambda^{2}-m^{2}}} K_{\lambda}^{*} K_{\lambda}
$$

if we use the operator

$$
K_{\lambda}: \mathcal{H} \rightarrow \mathrm{L}^{2}\left(\mathbb{R}^{2} ; \mathbb{C}^{8}\right), \quad K_{\lambda} \varphi:=\left(\begin{array}{l}
K_{1, \lambda} \varphi \\
K_{2, \lambda} \varphi
\end{array}\right)
$$

with adjoint

$$
K_{\lambda}^{*}: \mathrm{L}^{2}\left(\mathbb{R}^{2} ; \mathbb{C}^{8}\right) \rightarrow \mathcal{H}, \quad K_{\lambda}^{*}\left(\begin{array}{l}
\psi_{1} \\
\psi_{2}
\end{array}\right)=K_{1, \lambda}^{*} \psi_{1}+K_{2, \lambda}^{*} \psi_{2} .
$$

For the next proposition we also need to introduce for each $\lambda \in \mathbb{R}$ with $|\lambda|>m$ the positive operator $\Omega(\lambda): \mathrm{L}^{2}\left(\mathbb{R}^{2} ; \mathbb{C}^{8}\right) \rightarrow \mathrm{L}^{2}\left(\mathbb{R}^{2} ; \mathbb{C}^{8}\right)$ defined by

$$
\Omega(\lambda):=\frac{1}{2 \sqrt{\lambda^{2}-m^{2}}} K_{\lambda} K_{\lambda}^{*}
$$


A direct calculation shows that

$$
K_{\lambda} K_{\lambda}^{*}=p\left(\begin{array}{ll}
M_{1, \lambda} & M_{2, \lambda} \\
M_{2, \lambda} & M_{3, \lambda}
\end{array}\right) p
$$

where

$$
\begin{aligned}
& M_{1, \lambda}\left(x_{\perp}\right):=\int_{\mathbb{R}} \mathrm{d} x_{3} \cos ^{2}\left(x_{3} \sqrt{\lambda^{2}-m^{2}}\right)\left(\begin{array}{cccc}
|\lambda+m| V_{11}\left(x_{\perp}, x_{3}\right) & 0 & \sqrt{\lambda^{2}-m^{2}} V_{13}\left(x_{\perp}, x_{3}\right) & 0 \\
0 & 0 & 0 & 0 \\
\sqrt{\lambda^{2}-m^{2}} V_{31}\left(x_{\perp}, x_{3}\right) & 0 & |\lambda-m| V_{33}\left(x_{\perp}, x_{3}\right) & 0 \\
0 & 0 & 0 & 0
\end{array}\right),
\end{aligned}
$$

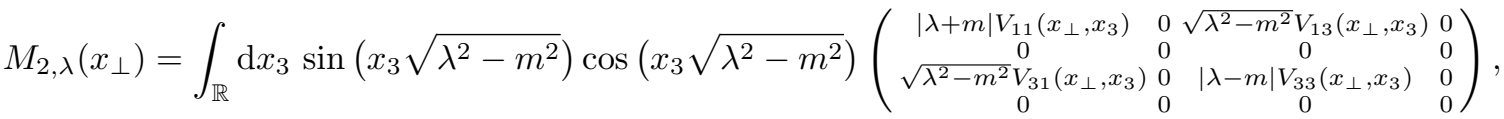

$$
\begin{aligned}
& M_{3, \lambda}\left(x_{\perp}\right)=\int_{\mathbb{R}} \mathrm{d} x_{3} \sin ^{2}\left(x_{3} \sqrt{\lambda^{2}-m^{2}}\right)\left(\begin{array}{cccc}
|\lambda+m| V_{11}\left(x_{\perp}, x_{3}\right) & 0 & \sqrt{\lambda^{2}-m^{2}} V_{13}\left(x_{\perp}, x_{3}\right) & 0 \\
0 & 0 & 0 & 0 \\
\sqrt{\lambda^{2}-m^{2}} V_{31}\left(x_{\perp}, x_{3}\right) & 0 & |\lambda-m| V_{33}\left(x_{\perp}, x_{3}\right) & 0 \\
0 & 0 & 0
\end{array}\right) .
\end{aligned}
$$

This implies that

$$
\|\Omega(\lambda)\|_{1} \leq\left(\frac{\lambda+m}{\lambda-m}\right)^{1 / 2}\left\|p W_{+} p\right\|_{1}+\left(\frac{\lambda-m}{\lambda+m}\right)^{1 / 2}\left\|p W_{-} p\right\|_{1},
$$

and thus $\Omega(\lambda) \in S_{1}\left[\mathrm{~L}^{2}\left(\mathbb{R}^{2} ; \mathbb{C}^{8}\right)\right]$ if $V$ satisfies Assumption 3.1.

Next Proposition is a direct consequence of Equations (6.16) and (6.25).

Proposition 6.13. Let $V$ satisfy Assumption 3.1. Then we have for each $\lambda \in \mathbb{R}$ with $|\lambda|>m$ and each $s>0$

$$
n_{ \pm}\left(s ; \operatorname{sgn}(\lambda) \operatorname{lm} T_{\text {div }}(\lambda)\right)=n_{ \pm}(s ; \Omega(\lambda)) .
$$

In particular, it follows by Equation (6.24) that

$$
\operatorname{Tr} \arctan \left(s^{-1} \operatorname{sgn}(\lambda) \operatorname{Im} T_{\text {div }}(\lambda)\right)=\operatorname{Tr} \arctan \left(s^{-1} \Omega(\lambda)\right) .
$$

The combination of Corollary 6.12 and Equation (6.26) gives the following.

Theorem 6.14. Let $V$ satisfy Assumption 3.2. Then one has for each $\varepsilon \in(0,1)$

$$
\pm \pi^{-1} \operatorname{Tr} \arctan \left[(1 \pm \varepsilon)^{-1} \Omega(\lambda)\right]+\mathcal{O}(1) \leq \xi\left(\lambda ; H_{ \pm}, H_{0}\right) \leq \pm \pi^{-1} \operatorname{Tr} \arctan \left[(1 \mp \varepsilon)^{-1} \Omega(\lambda)\right]+\mathcal{O}(1)
$$

as $\lambda \rightarrow \pm m,|\lambda|>m$.

Remark 6.15. The fact that the operators $\omega_{ \pm}(\lambda)$ and $\Omega(\lambda)$ in Theorems 6.5 and 6.14 depend in a distinguished way on the components $V_{11}$ and $V_{33}$ of $V$ is due to our initial assumption $b_{0}>0$. Indeed, this choice implies that $\operatorname{ker}\left(H_{\perp}^{-}\right)$is non trivial, whereas $\operatorname{ker}\left(H_{\perp}^{+}\right)=\{0\}$. This lead us to introduce in Section 5 the projection $\mathrm{P} \equiv \operatorname{diag}(P, 0, P, 0)$, which put into light the priviledged role of the components $V_{11}$ and $V_{33}$ of $V$.

The variation of $\xi\left(\lambda ; H_{ \pm}, H_{0}\right)$ under the change $\lambda \mapsto-\lambda$ can be explained using the antinunitary transformation of charge conjugation [38, Sec. 1.4.6]

$$
C: \mathcal{H} \rightarrow \mathcal{H}, \quad \varphi \mapsto U_{C} \bar{\varphi}
$$

where $U_{C}:=i \beta \alpha_{2}$. Indeed, if we write $H(\vec{a}, \pm V)$ and $H_{0}(\vec{a})$ for $H_{ \pm}$and $H_{0}$, then we get

$$
C H(\vec{a}, \pm V) C^{-1}=-H\left(-\vec{a}, \mp U_{C} \bar{V} U_{C}^{*}\right),
$$

and a direct calculation using the Lifshits-Krein trace formula (1.2) shows that

$$
\xi\left(\lambda ; H(\vec{a}, \pm V), H_{0}(\vec{a})\right)=-\xi\left(-\lambda ; H\left(-\vec{a}, \mp U_{C} \bar{V} U_{C}^{*}\right), H_{0}(-\vec{a})\right) .
$$

This obviously explains why the overall sign of the spectral shift function is reversed under the change $\lambda \mapsto$ $-\lambda$. But it also explains why the roles of $V_{11}$ and $V_{33}$ are interchanged in the estimates. Indeed, the natural projection corresponding to the vector potential $\vec{a}$ is $\mathrm{P}=\operatorname{diag}(P, 0, P, 0)$ since we have $b_{0}>0$ for $\vec{a}$, whereas 
$\mathrm{P}^{\prime}:=\operatorname{diag}(0, P, 0, P)$ is the natural choice for the vector potential $-\vec{a}$ since we have $b_{0}<0$ for $-\vec{a}$. Now, one has

$$
\mp U_{C} \bar{V} U_{C}^{*}=\mp\left(\begin{array}{cccc}
V_{44} & -\overline{V_{43}} & -\overline{V_{42}} & \overline{V_{41}} \\
-\overline{V_{34}} & \frac{V_{33}}{V_{23}} & -\overline{V_{31}} \\
-\overline{V_{24}} & -\overline{V_{13}} & -\overline{V_{12}} & -\overline{V_{21}}
\end{array}\right) .
$$

So, the projection $\mathrm{P}$ which selects the components $\pm\left(V_{11}, V_{33}\right)$ of the potential $\pm V$ is replaced, after the change $\lambda \mapsto-\lambda$, by the projection $\mathrm{P}^{\prime}$ which selects the components $\mp\left(V_{33}, V_{11}\right)$ of the transformed potential $\mp U_{C} \bar{V} U_{C}^{*}$.

For the next proposition we define for each $\lambda \in \mathbb{R}$ with $|\lambda|>m$ the positive operator $\Omega^{(1)}(\lambda)$ in $L^{2}\left(\mathbb{R}^{2} ; \mathbb{C}^{8}\right)$ given by

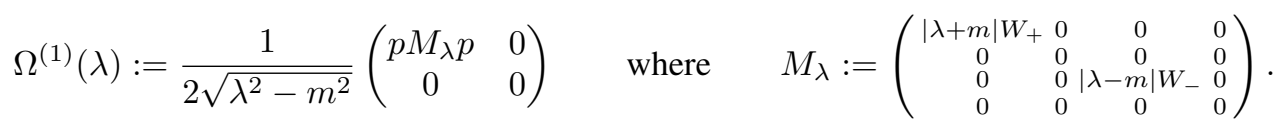

Proposition 6.16. (a) Let $V$ satisfy Assumption 3.2 with $\nu \in(3,4]$. Then one has for each $s>0$ and each $\delta \in\left(\frac{4-\nu}{2}, \frac{1}{2}\right)$

$$
\operatorname{Tr} \arctan \left[s^{-1} \Omega(\lambda)\right]-\operatorname{Tr} \arctan \left[s^{-1} \Omega^{(1)}(\lambda)\right]=O\left(|\lambda \mp m|^{-\delta}\right) \quad \text { as } \quad \lambda \rightarrow \pm m,|\lambda|>m .
$$

(b) Let $V$ satisfy Assumption 3.1 with $\nu_{\perp}>2$ and $\nu_{3}>2$. Then one has for each $s>0$

$$
\operatorname{Tr} \arctan \left[s^{-1} \Omega(\lambda)\right]-\operatorname{Tr} \arctan \left[s^{-1} \Omega^{(1)}(\lambda)\right]=\mathcal{O}(1) \quad \text { as } \quad \lambda \rightarrow \pm m,|\lambda|>m .
$$

Proof. Points (a) and (b) are proved by using the Lifshits-Krein trace formula (1.2) with $f(\lambda)=\arctan (\lambda)$, $\lambda \in \mathbb{R}$. We do not give the details, since the argument is analogous to the one of [12, Cor. 2.2].

Note that if $V$ satisfy Assumption 3.2 with $\nu \in(3,4]$, we can choose $\delta \in\left(\frac{4-\nu}{2}, \frac{1}{\nu-1}\right)$, and so Proposition 6.16.(a) entails

$$
\operatorname{Tr} \arctan \left[s^{-1} \Omega(\lambda)\right]-\operatorname{Tr} \arctan \left[s^{-1} \Omega^{(1)}(\lambda)\right]=o\left(|\lambda \mp m|^{-\frac{1}{\nu-1}}\right) \quad \text { as } \quad \lambda \rightarrow \pm m,|\lambda|>m .
$$

Moreover, if $V$ satisfy Assumption 3.2 with $\nu>4$, then it satisfies Assumption 3.1 with $\nu_{\perp}>2$ and $\nu_{3}>2$, and, hence (6.27) is valid. Finally, we have for $s>0$ and $|\lambda|>m$

$\operatorname{Tr} \arctan \left[s^{-1} \Omega^{(1)}(\lambda)\right]=\int_{0}^{\infty} \frac{\mathrm{d} t}{1+t^{2}} n_{+}\left(2 s t\left(\frac{\lambda-m}{\lambda+m}\right)^{1 / 2} ; p W_{+} p\right)+\int_{0}^{\infty} \frac{\mathrm{d} t}{1+t^{2}} n_{+}\left(2 s t\left(\frac{\lambda+m}{\lambda-m}\right)^{1 / 2} ; p W_{-} p\right)$.

Combining Equations (6.27)-(6.29), Theorem 6.14 and Lemmas 6.7-6.9, we get the following.

Corollary 6.17. Let V satisfy Assumption 3.2.

(a) Assume that the hypotheses of Lemma 6.7 hold with $U_{ \pm}=W_{ \pm}$and $\alpha=\nu-1$. Then we have

$$
\xi\left(\lambda ; H_{-}, H_{0}\right)=-\frac{1}{2 \cos (\pi /(\nu-1))} \Psi_{\nu-1}\left(2\left(\frac{\lambda-m}{\lambda+m}\right)^{1 / 2} ; u_{+}, b_{0}\right)(1+o(1)) \quad \text { as } \quad \lambda \searrow m,
$$

and

$$
\xi\left(\lambda ; H_{+}, H_{0}\right)=\frac{1}{2 \cos (\pi /(\nu-1))} \Psi_{\nu-1}\left(2\left(\frac{\lambda+m}{\lambda-m}\right)^{1 / 2} ; u_{-}, b_{0}\right)(1+o(1)) \quad \text { as } \quad \lambda \nearrow-m,
$$

with $\Psi_{\nu-1}$ given by Equation (6.21). 
(b) Suppose that $V$ also satisfies (3.1) with $\nu_{\perp}>2$ and $\nu_{3}>2$, and assume that the hypotheses of Lemma 6.8 hold with $U_{ \pm}=W_{ \pm}$. Then we have

$$
\xi\left(\lambda ; H_{-}, H_{0}\right)=-\frac{1}{2} \Phi_{\beta_{+}}\left(2\left(\frac{\lambda-m}{\lambda+m}\right)^{1 / 2} ; \eta_{+}, b_{0}\right)(1+o(1)) \quad \text { as } \quad \lambda \searrow m,
$$

and

$$
\xi\left(\lambda ; H_{-}, H_{0}\right)=\frac{1}{2} \Phi_{\beta_{-}}\left(2\left(\frac{m+\lambda}{m-\lambda}\right)^{1 / 2} ; \eta_{-}, b_{0}\right)(1+o(1)) \text { as } \lambda \nearrow-m,
$$

with $\beta_{ \pm} \in(0, \infty)$ and $\Phi_{\beta_{ \pm}}$given by Equation (6.22).

(c) Suppose that $V$ also satisfies (3.1) with $\nu_{\perp}>2$ and $\nu_{3}>2$, and assume that the hypotheses of Lemma 6.9 hold with $U_{ \pm}=W_{ \pm}$. Then we have

$$
\xi\left(\lambda ; H_{-}, H_{0}\right)=-\frac{1}{2} \Phi_{\infty}\left(2\left(\frac{m-\lambda}{m+\lambda}\right)^{1 / 2}\right)(1+o(1)) \quad \text { as } \quad \lambda \searrow m
$$

and

$$
\xi\left(\lambda ; H_{+}, H_{0}\right)=\frac{1}{2} \Phi_{\infty}\left(2\left(\frac{m+\lambda}{m-\lambda}\right)^{1 / 2}\right)(1+o(1)) \text { as } \lambda \nearrow-m,
$$

with $\Phi_{\infty}$ given by Equation (6.23).

Putting together the results of Proposition 6.10 and Corollary 6.17, we obtain the following.

Corollary 6.18. Under the assumptions of Corollary 6.17.(a), we have

$$
\lim _{\varepsilon \searrow 0} \frac{\xi\left(m(1-\varepsilon)^{-1} ; H_{-}, H_{0}\right)}{\xi\left(m(1-\varepsilon) ; H_{-}, H_{0}\right)}=\frac{1}{2 \cos (\pi /(\nu-1))}=\lim _{\varepsilon \searrow 0} \frac{\xi\left(-m(1-\varepsilon)^{-1} ; H_{+}, H_{0}\right)}{\xi\left(-m(1-\varepsilon) ; H_{+}, H_{0}\right)},
$$

and under the assumptions of Corollary 6.17.(b)-(c), we have

$$
\lim _{\varepsilon \searrow 0} \frac{\xi\left(m(1-\varepsilon)^{-1} ; H_{-}, H_{0}\right)}{\xi\left(m(1-\varepsilon) ; H_{-}, H_{0}\right)}=\frac{1}{2}=\lim _{\varepsilon \searrow 0} \frac{\xi\left(-m(1-\varepsilon)^{-1} ; H_{+}, H_{0}\right)}{\xi\left(-m(1-\varepsilon) ; H_{+}, H_{0}\right)} .
$$

\section{Acknowledgements}

The author expresses his deep gratitude to Professor G. D. Raikov for suggesting him this study. He also thanks him for the idea of using the decomposition (5.1) for the free Hamiltonian.

\section{Appendix}

We give in this appendix the proof of the inclusion (3.6) for the class of potentials $V$ given in Remark 3.3. We start with a technical lemma. We use the notations $\alpha:=\left(\alpha_{1}, \alpha_{2}, \alpha_{3}\right)^{\top}$ and

$$
\left(\partial_{\ell} V\right):=\left\{\left(\partial_{\ell} V_{j k}\right)\right\}, \quad \nabla V:=\left(\partial_{1} V, \partial_{2} V, \partial_{3} V\right)^{\top}, \quad\left(\partial_{\ell} \partial_{m} V\right):=\left\{\left(\partial_{\ell} \partial_{m} V_{j k}\right)\right\} .
$$

Lemma 7.1. Let $V$ be as in Remark 3.3. Then

(a) One has in $\mathscr{B}\left(\mathcal{D}\left(H_{0}\right), \mathcal{D}\left(H_{0}\right)^{*}\right)$ the equalities

$$
\begin{aligned}
{\left[H_{0}, H\right] } & =-i \alpha \cdot(\nabla V)+\left[\alpha_{3}, V\right] P_{3}+m[\beta, V] \\
& =-i \alpha \cdot(\nabla V)+P_{3}\left[\alpha_{3}, V\right]+i\left[\alpha_{3},\left(\partial_{3} V\right)\right]+m[\beta, V] .
\end{aligned}
$$

(b) Let $z \in \mathbb{R} \backslash\left\{\sigma\left(H_{0}\right) \cup \sigma\left(H_{ \pm}\right)\right\}$. Then there exist operators $B_{ \pm} \in \mathscr{B}(\mathcal{H})$ such that

$$
R_{ \pm}^{2}(z)=B_{ \pm} H_{0}^{-2} \quad \text { and } \quad R_{ \pm}^{2}(z)=H_{0}^{-2} B_{ \pm}^{*} .
$$


Proof. (a) We know from Lemma 2.2(b) of [32] that $\mathcal{D}\left(H_{0}\right) \subset \mathcal{D}\left(P_{3}\right)$. So each member of Equations (7.1)-(7.2) belongs to $\mathscr{B}\left(\mathcal{D}\left(H_{0}\right), \mathcal{D}\left(H_{0}\right)^{*}\right)$.

Let $\varphi \in \mathcal{D}\left(H_{0}\right)$, take a sequence $\left\{\varphi_{n}\right\} \subset C_{0}^{\infty}\left(\mathbb{R}^{3} ; \mathbb{C}^{4}\right)$ such that $\lim _{n}\left\|\varphi_{n}-\varphi\right\|_{\mathcal{D}\left(H_{0}\right)}=0$, and denote by $\langle\cdot, \cdot\rangle_{1,-1}$ the anti-duality map between $\mathcal{D}\left(H_{0}\right)$ and $\mathcal{D}\left(H_{0}\right)^{*}$. Then

$$
\begin{aligned}
\left\langle\varphi,\left[H_{0}, H\right] \varphi\right\rangle_{1,-1} & \equiv\left\langle H_{0} \varphi, V \varphi\right\rangle-\left\langle V \varphi, H_{0} \varphi\right\rangle \\
& =\lim _{n}\left\langle\varphi_{n},\left[\alpha_{1}\left(P_{1}-a_{1}\right)+\alpha_{2}\left(P_{2}-a_{2}\right)+\alpha_{3} P_{3}+\beta m, V\right] \varphi_{n}\right\rangle \\
& =\lim _{n}\left\langle\varphi_{n},\left\{-i \alpha \cdot(\nabla V)+\left[\alpha_{3}, V\right] P_{3}+m[\beta, V]\right\} \varphi_{n}\right\rangle .
\end{aligned}
$$

Since $\mathcal{D}\left(H_{0}\right) \subset \mathcal{D}\left(P_{3}\right)$, we also have $\lim _{n}\left\|\varphi_{n}-\varphi\right\|_{\mathcal{D}\left(P_{3}\right)}=0$, and thus

$$
\left\langle\varphi,\left[H_{0}, H\right] \varphi\right\rangle_{1,-1}=\left\langle\varphi,\left\{-i \alpha \cdot(\nabla V)+\left[\alpha_{3}, V\right] P_{3}+m[\beta, V]\right\} \varphi\right\rangle
$$

This proves (7.1). Using (7.4), one also gets the equality (7.2).

(b) In what follows, we omit the indices " \pm " to simplify the notations and we write $B_{1}, B_{2}, \ldots$ for elements of $\mathscr{B}(\mathcal{H})$. Since $\mathcal{D}(H)=\mathcal{D}\left(H_{0}\right)$, we have

$$
R^{2}(z)=B_{1} H_{0}^{-1} R(z)=B_{1} R(z) H_{0}^{-1}+B_{1}\left[H_{0}^{-1}, R(z)\right]=B_{2} H_{0}^{-2}+B_{1} H_{0}^{-1} R(z)\left[H_{0}, H\right] R(z) H_{0}^{-1} .
$$

Now, one has

$$
R(z)\left[H_{0}, H\right] R(z) H_{0}^{-1}=R(z)\left\{-i \alpha \cdot(\nabla V)+P_{3}\left[\alpha_{3}, V\right]+i\left[\alpha_{3},\left(\partial_{3} V\right)\right]+m[\beta, V]\right\} R(z) H_{0}^{-1}=B_{3} H_{0}^{-2}
$$

due to Equation (7.2), the equality $\mathcal{D}(H)=\mathcal{D}\left(H_{0}\right)$, and the inclusion $\mathcal{D}\left(H_{0}\right) \subset \mathcal{D}\left(P_{3}\right)$. This, together with the preceding equation, implies the first identity in (7.3). The second identity follows from the first one by adjunction.

Proposition 7.2. Take $z \in \mathbb{R} \backslash\left\{\sigma\left(H_{0}\right) \cup \sigma\left(H_{ \pm}\right)\right\}$and let $V$ be as in Remark 3.3. Then we have

$$
R_{ \pm}^{3}(z)-R_{0}^{3}(z) \in S_{1}(\mathcal{H}) .
$$

Proof. In what follows, we omit the indices " \pm " to simplify the notations and we write $B_{1}, B_{2}, \ldots$ for elements of $\mathscr{B}(\mathcal{H})$. Differentiating twice the resolvent identity

$$
R(z)-R_{0}(z)=-R(z) V R_{0}(z)
$$

we find that

$$
R^{3}(z)-R_{0}^{3}(z)=-R(z) V R_{0}^{3}(z)-R^{2}(z) V R_{0}^{2}(z)-R^{3}(z) V R_{0}(z) .
$$

So it is sufficient to show that each term on the r.h.s. belongs to $S_{1}(\mathcal{H})$. This is done in points (i), (ii) and (iii) below.

(i) For the term $R(z) V R_{0}^{3}(z)$, one has

$$
R(z) V R_{0}^{3}(z)=R(z) R_{0}(z) V R_{0}^{2}(z)+R(z)\left[V, R_{0}(z)\right] R_{0}^{2}(z) .
$$

Since $\mathcal{D}(H)=\mathcal{D}\left(H_{0}\right)$, one has

$$
R(z) R_{0}(z) V R_{0}^{2}(z)=R(z)\left(H_{0}-z\right) R_{0}^{2}(z) V R_{0}^{2}(z)=\left(B_{1} H_{0}^{-2} V^{1 / 2}\right)\left(V^{1 / 2} H_{0}^{-2} B_{2}\right) .
$$

So, by (3.3), $R(z) R_{0}(z) V R_{0}^{2}(z)$ is the product of two Hilbert-Schmidt operators, and thus belongs to $S_{1}(\mathcal{H})$.

For the second term of (7.5), we have by (7.1)

$$
R(z)\left[V, R_{0}(z)\right] R_{0}^{2}(z)=R(z) R_{0}(z)\left[H_{0}, H\right] R_{0}^{3}(z)=B_{1} H_{0}^{-2}\left\{-i \alpha \cdot(\nabla V)+\left[\alpha_{3}, V\right] P_{3}+m[\beta, V]\right\} H_{0}^{-3} B_{3} .
$$


Due to the hypotheses on $V$ and $\left(\partial_{j} V\right)$, one can use (3.3) to write the first and third term as a product of two Hilbert-Schmidt operators. So it only remains to show that $H_{0}^{-2}\left[\alpha_{3}, V\right] P_{3} H_{0}^{-3}$ belongs to $S_{1}(\mathcal{H})$. For this, we use the inclusion $\mathcal{D}\left(H_{0}\right) \subset \mathcal{D}\left(P_{3}\right)$ and the commutation of $P_{3}$ and $H_{0}^{-1}$ on $\mathcal{D}\left(P_{3}\right)$ [32, Lemma 2.2(b)] to get

$$
H_{0}^{-2}\left[\alpha_{3}, V\right] P_{3} H_{0}^{-3}=H_{0}^{-2}\left[\alpha_{3}, V\right] H_{0}^{-2} P_{3} H_{0}^{-1}=H_{0}^{-2}\left[\alpha_{3}, V\right] H_{0}^{-2} B_{4} .
$$

This, together with (3.3), implies that $H_{0}^{-2}\left[\alpha_{3}, V\right] P_{3} H_{0}^{-3}$ belongs to $S_{1}(\mathcal{H})$.

(ii) One can write $R^{2}(z) V R_{0}^{2}(z)$ as the product of two Hilbert-Schmidt operators by using (7.3) and (3.3):

$$
R^{2}(z) V R_{0}^{2}(z)=B_{5} H_{0}^{-2} V H_{0}^{-2} B_{2}=\left(B_{5} H_{0}^{-2} V^{1 / 2}\right)\left(V^{1 / 2} H_{0}^{-2} B_{2}\right) .
$$

Thus $R^{2}(z) V R_{0}^{2}(z)$ belongs to $S_{1}(\mathcal{H})$.

(iii) For the term $R^{3}(z) V R_{0}(z)$ we have

$$
R^{3}(z) V R_{0}(z)=R^{2}(z) V R(z) R_{0}(z)+R^{2}(z)[R(z), V] R_{0}(z) .
$$

One shows that $R^{2}(z) V R(z) R_{0}(z) \in S_{1}(\mathcal{H})$ as in point (ii). For the second term, we have by (7.2) and (7.3)

$$
\begin{aligned}
R^{2}(z)[R(z), V] R_{0}(z) & =R^{3}(z)\left[H, H_{0}\right] R(z) R_{0}(z) \\
& =B_{5} H_{0}^{-2} R(z)\left\{i \alpha \cdot(\nabla V)-P_{3}\left[\alpha_{3}, V\right]-i\left[\alpha_{3},\left(\partial_{3} V\right)\right]-m[\beta, V]\right\} H_{0}^{-2} B_{6} .
\end{aligned}
$$

Due to the hypotheses on $V$ and $\left(\partial_{j} V\right)$, one can use (7.3) and (3.3) to write the first, third, and fourth term as a product of two Hilbert-Schmidt operators. So it only remains to show that $H_{0}^{-2} R(z) P_{3}\left[\alpha_{3}, V\right] H_{0}^{-2}$ belongs to $S_{1}(\mathcal{H})$. Using [32, Lemma 2.2(b)] and (7.3), one gets

$$
\begin{aligned}
H_{0}^{-2} R(z) P_{3}\left[\alpha_{3}, V\right] H_{0}^{-2} & =H_{0}^{-2} P_{3} R(z)\left[\alpha_{3}, V\right] H_{0}^{-2}+H_{0}^{-2}\left[R(z), P_{3}\right]\left[\alpha_{3}, V\right] H_{0}^{-2} \\
& =P_{3} H_{0}^{-2} R(z)\left[\alpha_{3}, V\right] H_{0}^{-2}-i H_{0}^{-2} R(z)\left(\partial_{3} V\right) R(z)\left[\alpha_{3}, V\right] H_{0}^{-2} \\
& =B_{7} H_{0}^{-2}\left[\alpha_{3}, V\right] H_{0}^{-2}+B_{8} R(z)\left(\partial_{3} V\right) R(z)\left[\alpha_{3}, V\right] H_{0}^{-2} .
\end{aligned}
$$

The first term on the r.h.s. belongs to $S_{1}(\mathcal{H})$, and for the second term we have by (7.2) and (7.3)

$$
\begin{aligned}
& R(z)\left(\partial_{3} V\right) R(z)\left[\alpha_{3}, V\right] H_{0}^{-2} \\
& \begin{aligned}
=\left(\partial_{3} V\right) R^{2}(z)\left[\alpha_{3}, V\right] H_{0}^{-2}+R(z)[ & \left.\left(\partial_{3} V\right), H\right] R^{2}(z)\left[\alpha_{3}, V\right] H_{0}^{-2} \\
=B_{9} H_{0}^{-2}\left[\alpha_{3}, V\right] H_{0}^{-2}+R(z)\{i \alpha \cdot & {\left[\nabla\left(\partial_{3} V\right)\right]-P_{3}\left[\alpha_{3},\left(\partial_{3} V\right)\right] } \\
& \left.\quad-i\left[\alpha_{3},\left(\partial_{3}^{2} V\right)\right]-m\left[\beta,\left(\partial_{3} V\right)\right]+\left[\left(\partial_{3} V\right), V\right]\right\} B_{5} H_{0}^{-2}\left[\alpha_{3}, V\right] H_{0}^{-2} .
\end{aligned}
\end{aligned}
$$

Due to the hypotheses on $V,\left(\partial_{j} V\right)$, and $\left(\partial_{j 3} V\right)$, one can use (3.3) to show that the first, the second, the fourth, the fifth, and the sixth term are trace class. For the third term we have to use (3.3) and the fact that $R(z) P_{3}$ extends to a bounded operator.

Remark 7.3. When the potential $V$ is scalar, the equations (7.1)-(7.2) reduce to the single equality

$$
\left[H_{0}, H\right]=-i \alpha \cdot(\nabla V)
$$

in $\mathscr{B}\left(\mathcal{D}\left(H_{0}\right), \mathcal{D}\left(H_{0}\right)^{*}\right)$. So the calculations in points (i) and (iii) of the proof of Proposition 7.2 simplify accordingly, and we obtain the inclusion

$$
R_{ \pm}^{3}(z)-R_{0}^{3}(z) \in S_{1}(\mathcal{H})
$$

without assuming anything on the derivatives of $V$ of order 2. 


\section{References}

[1] J. Avron, I. Herbst, and B. Simon. Schrödinger operators with magnetic fields. I. General interactions. Duke Math. J., 45(4):847-883, 1978.

[2] A. Berthier and V. Georgescu. On the point spectrum of Dirac operators. J. Funct. Anal., 71(2):309-338, 1987.

[3] M. Sh. Birman and T. A. Suslina. The periodic Dirac operator is absolutely continuous. Integral Equations Operator Theory, 34(4):377-395, 1999.

[4] N. Boussaid and S. Golénia. Limiting absorption principle for some long range perturbations of Dirac systems at threshold energies. preprint on http://arxiv.org/abs/0906.1495.

[5] A. Boutet de Monvel-Berthier, D. Manda, and R. Purice. Limiting absorption principle for the Dirac operator. Ann. Inst. H. Poincaré Phys. Théor., 58(4):413-431, 1993.

[6] V. Bruneau, A. Pushnitski, and G. Raikov. Spectral shift function in strong magnetic fields. Algebra $i$ Analiz, 16(1):207-238, 2004.

[7] V. Bruneau and D. Robert. Asymptotics of the scattering phase for the Dirac operator: high energy, semiclassical and non-relativistic limits. Ark. Mat., 37(1):1-32, 1999.

[8] P. A. Cojuhari. On the finiteness of the discrete spectrum of the Dirac operator. Rep. Math. Phys., 57(3):333-341, 2006.

[9] E. B. Davies. Linear operators and their spectra, volume 106 of Cambridge Studies in Advanced Mathematics. Cambridge University Press, Cambridge, 2007.

[10] S. Doi, A. Iwatsuka, and T. Mine. The uniqueness of the integrated density of states for the Schrödinger operators with magnetic fields. Math. Z., 237(2):335-371, 2001.

[11] W. D. Evans and R. T. Lewis. Eigenvalue estimates in the semi-classical limit for Pauli and Dirac operators with a magnetic field. R. Soc. Lond. Proc. Ser. A Math. Phys. Eng. Sci., 455(1981):183-217, 1999.

[12] C. Fernández and G. Raikov. On the singularities of the magnetic spectral shift function at the Landau levels. Ann. Henri Poincaré, 5(2):381-403, 2004.

[13] V. Georgescu and M. Măntoiu. On the spectral theory of singular Dirac type Hamiltonians. J. Operator Theory, 46(2):289-321, 2001.

[14] F. Gesztesy and K. A. Makarov. The $\Xi$ operator and its relation to Krein's spectral shift function. J. Anal. Math., 81:139-183, 2000.

[15] A. Grigis and A. Mohamed. Finitude des lacunes dans le spectre de l'opérateur de Schrödinger et de celui de Dirac avec des potentiels électrique et magnétique périodiques. J. Math. Kyoto Univ., 33(4):1071-1096, 1993.

[16] G. Hachem. Effet Zeeman pour un électron de Dirac. Ann. Inst. H. Poincaré Phys. Théor., 58(1):105-123, 1993.

[17] B. Helffer, J. Nourrigat, and X. P. Wang. Sur le spectre de l'équation de Dirac (dans $\mathbb{R}^{2}$ ou $\mathbb{R}^{3}$ ) avec champ magnétique. Ann. scient. Éc. Norm. Sup., 22:515-533, 1989.

[18] T. Hupfer, H. Leschke, P. Müller, and S. Warzel. Existence and uniqueness of the integrated density of states for Schrödinger operators with magnetic fields and unbounded random potentials. Rev. Math. Phys., 13(12):1547-1581, 2001. 
[19] A. Iftimovici and M. Măntoiu. Limiting absorption principle at critical values for the Dirac operator. Lett. Math. Phys., 49(3):235-243, 1999.

[20] V. Ivrii. Microlocal analysis and precise spectral asymptotics. Springer Monographs in Mathematics. Springer-Verlag, Berlin, 1998.

[21] M. Klaus. On the Levinson theorem for Dirac operators. J. Math. Phys., 31(1):182-190, 1990.

[22] Z.-Q. Ma. The Levinson theorem. J. Phys. A, 39(48):R625-R659, 2006.

[23] M. Melgaard and G. Rozenblum. Eigenvalue asymptotics for weakly perturbed Dirac and Schrödinger operators with constant magnetic fields of full rank. Comm. Partial Differential Equations, 28(3-4):697736, 2003.

[24] G. J. Murphy. $C^{*}$-algebras and operator theory. Academic Press Inc., Boston, MA, 1990.

[25] A. Pushnitski. The spectral shift function and the invariance principle. J. Funct. Anal., 183(2):269-320, 2001.

[26] A. B. Pushnitski1. A representation for the spectral shift function in the case of perturbations of fixed sign. Algebra i Analiz, 9(6):197-213, 1997.

[27] G. D. Raikov. Low Energy Asymptotics of the SSF for Pauli Operators with Nonconstant Magnetic Fields. preprint on http://arxiv.org/abs/0908.3704.

[28] G. D. Raikov. Eigenvalue asymptotics for the Dirac operator in strong constant magnetic fields. Math. Phys. Electron. J., 5:Paper 2, 22 pp. (electronic), 1999.

[29] G. D. Raikov. Spectral asymptotics for the perturbed 2D Pauli operator with oscillating magnetic fields. I. Non-zero mean value of the magnetic field. Markov Process. Related Fields, 9(4):775-794, 2003.

[30] G. D. Raikov and S. Warzel. Quasi-classical versus non-classical spectral asymptotics for magnetic Schrödinger operators with decreasing electric potentials. Rev. Math. Phys., 14(10):1051-1072, 2002.

[31] M. Reed and B. Simon. Methods of modern mathematical physics. III. Academic Press [Harcourt Brace Jovanovich Publishers], New York, 1979. Scattering theory.

[32] S. Richard and R. Tiedra de Aldecoa. On perturbations of Dirac operators with variable magnetic field of constant direction. J. Math. Phys., 45(11):4164-4173, 2004.

[33] S. Richard and R. Tiedra de Aldecoa. On the spectrum of magnetic Dirac operators with Coulomb-type perturbations. J. Funct. Anal., 250(2):625-641, 2007.

[34] D. Robert. Semiclassical asymptotics for the spectral shift function. In Differential operators and spectral theory, volume 189 of Amer. Math. Soc. Transl. Ser. 2, pages 187-203. Amer. Math. Soc., Providence, RI, 1999.

[35] O. Safronov. Spectral shift function in the large coupling constant limit. J. Funct. Anal., 182(1):151-169, 2001.

[36] Y. Saito and T. Umeda. Eigenfunctions at the threshold energies of magnetic Dirac operators. preprint on http://arxiv.org/abs/0905.0961.

[37] B. Thaller. Dirac particles in magnetic fields. In Recent developments in quantum mechanics (Poiana Braşov, 1989), volume 12 of Math. Phys. Stud., pages 351-366. Kluwer Acad. Publ., Dordrecht, 1991.

[38] B. Thaller. The Dirac Equation. Springer-Verlag, Berlin, 1992. 
[39] D. R. Yafaev. Mathematical scattering theory, volume 105 of Translations of Mathematical Monographs. American Mathematical Society, Providence, RI, 1992. General theory, Translated from the Russian by J. R. Schulenberger.

[40] D. R. Yafaev. A trace formula for the Dirac operator. Bull. London Math. Soc., 37(6):908-918, 2005. 